Pacific

Journal of

Mathematics

\title{
BRAID COMMUTATORS AND HOMOGENOUS CAMPBELL-HAUSDORFF TESTS
}

Stefan Papadima 


\title{
BRAID COMMUTATORS AND HOMOGENOUS CAMPBELL-HAUSDORFF TESTS
}

\author{
STEFAN PAPADIMA
}

\begin{abstract}
We develop our general machinery of the Campbell-Hausdorff invariants of links, in the case of pure links, with emphasis on the connections with the lower central series of the pure braid groups. We present a complete simple set of rules for the Artin calculus of longitudes modulo the central series. We prove that if two pure links differ by an order $k$ pure braid commutator, then their order $k$ Campbell-Hausdorff invariants $p^{(k)}$ are the same. In this case, the general theory offers a decision test for the equality of $p^{(k+1)}$-invariants. We introduce the notion of homogenous link, which leads to important computational improvements for the general $p^{(k+1)}$-test. We provide both general homogeneity criteria and concrete interesting classes of homogenous examples. We illustrate the efficiency of our approach, on several classes of examples which cannot be distinguished by other known link invariants.
\end{abstract}

\section{Introduction}

This paper is a sequel to [22], where we have introduced a sequence, $\left\{p^{(k)}\right\}_{k \geq 2}$, of so-called Campbell-Hausdorff invariants of links. Let $K$ be an oriented and ordered $n$-component tame link in $S^{3}$, with fundamental group $G=: \pi_{1}\left(S^{3} \backslash K\right)$.

Our starting point is as in Milnor's construction of the $\bar{\mu}$-invariants [19]. The group $G$ comes naturally equipped with a geometric peripheral structure, consisting of $n$ meridians, $m_{i} \in G$, and $n$ longitudes, $l_{i} \in G$, associated to the components of $K$, for $i=1, \ldots, n$. For each fixed $k, k \geq 2$, Milnor noticed that the meridians generate the nilpotent quotient $G / \Gamma_{k} G$, where $\Gamma_{k} G$ stands for the $k$-fold commutators of the group $G$. Consequently, one may express each longitude (modulo commutators of length $k$ ) as a free word in the meridians, $l_{i}^{(k)} \in \mathbf{F}_{X}$, for $i=1, \ldots, n$. Here $\mathbf{F}_{X}$ denotes the free group generated by $x_{1}, \ldots, x_{n}$. In this way, one gets a system of algebraic longitudes, $\underline{l}=:\left\{l_{1}, \ldots, l_{n}\right\}$, where $l_{i}=\left\{l_{i}^{(k)}\right\}_{k \geq 2}$, for $i=1, \ldots, n$, satisfying certain convergence and normalization conditions. See [19].

Milnor's idea was to extract from $\underline{l}$ numerical invariants for $K$, by looking at the various (integer) coefficients of the Magnus expansions of the free 
words $l_{i}^{(k)}$. He was led to actually consider certain residue classes of these coefficients, in order to eliminate the indeterminacy created by the various possible choices for $\underline{l}$. One serious drawback is the fact that each $\bar{\mu}$-invariant takes values in a cyclic group which depends on the given link $K$.

Our basic idea in [22] was to introduce a new universal indeterminacy, which depends only on the number of components, $n$. We are going to recall it, briefly; see the next section for details. This indeterminacy is encoded by the action of a certain pro-unipotent $\mathbf{Q}$-group, $U$, on a certain filtered Q-vector space consisting of formal Lie derivations, $\operatorname{Der}_{1}^{+}$, filtered by $\left\{F_{k} \operatorname{Der}_{1}^{+}\right\}_{k}$. The $U$-action on $\operatorname{Der}_{1}^{+}$is $\mathbf{Q}$-linear and filtration-preserving, and everything depends only on $n$. The Campbell-Hausdorff expansion may be used to construct a derivation, $\partial(\underline{l}) \in \operatorname{Der}_{1}^{+}$, for any given system of algebraic longitudes, $\underline{l}$. For each $k \geq 2$, the $k$-th $C H$-invariant of $K$, $p^{(k)}(K) \in U \backslash \operatorname{Der}_{1}^{+} / F_{k} \operatorname{Der}_{1}^{+}$, is, by definition, the $U$-orbit of $\partial(\underline{l})$ modulo $F_{k}$ Der $_{1}^{+}$. We have proved in $\left[\mathbf{2 2}\right.$, Theorem A] that $p^{(k)}(K)$ is a well-defined concordance invariant of $K$, for all $k$.

In more geometric terms, our $C H$ approach has the following interpretation. Each nilpotent quotient, $G / \Gamma_{k} G$, inherits from the link complement a natural homological marking; that is, a collection of $2 n$ distinguished homology classes in degrees 1 and 2, given by the orientation classes of the meridians and neighbouring 2-tori respectively, corresponding to the components of the link. This homological marking passes to the Malcev completion, $\left(G / \Gamma_{k} G\right) \otimes \mathbf{Q}$. Let $K$ and $K^{\prime}$ be two $n$-component links, with fundamental groups $G$ and $G^{\prime}$. We have shown in [22, Remark 2.9] that $p^{(k)}(K)=p^{(k)}\left(K^{\prime}\right)$ if and only if the groups $\left(G / \Gamma_{k} G\right) \otimes \mathbf{Q}$ and $\left(G^{\prime} / \Gamma_{k} G^{\prime}\right) \otimes \mathbf{Q}$ are isomorphic by an isomorphism which preserves their distinguished lowdimensional homology classes.

The two approaches lead to the same primary invariants; see [22, Remark 2.7]. Note however that in general the $\bar{\mu}$-invariants and the $C H$-invariants exhibit an independent behaviour, as pointed out in [22, Example 3.4].

Our main goal in this paper is to develop and improve the general $\mathrm{CH}$ scheme, for the case of pure links. We are going to view the oriented links as Artin closures, $\widehat{b}$, of elements $b \in B_{n}$, where $B_{n}$ denotes Artin's braid group on $n$ strings. If $b \in P_{n} \subset B_{n}$ (where $P_{n}$ is the pure braid group on $n$ strings), then one knows that $\widehat{b}$ has $n$ components, which are naturally oriented and ordered. One good reason for looking at pure links is the existence of a canonical simple system of algebraic longitudes, to be denoted by $\underline{l}(b)=\left\{l_{1}(b), \ldots, l_{n}(b)\right\}$, for any pure link, $\widehat{b}$. It has the property that $l_{i}^{(k)}(\widehat{b})=l_{i}(b)$, for all $k$, and it can be canonically computed, by using the embedding, $B_{n} \subset \operatorname{Aut}\left(\mathbf{F}_{X}\right)$, provided by the Artin representation. See for example $[\mathbf{3}, \mathbf{2 1}]$, and also the next section, for details. The corresponding $C H$-invariants of $\widehat{b}$ will be denoted by $\left\{p^{(k)}(b)\right\}$. 
Like the $\bar{\mu}$-invariants, the $C H$-invariants are sensitive enough to detect the unlink among pure links, and also to detect the lower central series filtration of $P_{n},\left\{\Gamma_{k} P_{n}\right\}$. See Remark 1.1, and Corollary 2.9 respectively, for the precise statements, in a slightly more general (unordered and unoriented) form.

We are thus going to look at pairs of pure braids of the form $b=\beta \alpha$ and $b^{\prime}=\beta^{\prime} \alpha$, where $\alpha \in P_{n}$ and $\beta, \beta^{\prime} \in \Gamma_{k} P_{n}$, with $k \geq 2$. The relation between the $C H$-invariants and the lower central series of the pure braid groups will be a recurrent theme of this paper. It was suggested by a result of Stanford [24, Theorem 1] in the theory of finite-type (Vassiliev) invariants of links. Stanford's result says that if $b$ and $b^{\prime}$ are as above, then $\widehat{b}$ and $\widehat{b^{\prime}}$ have the same Vassiliev invariants, up to order $k-1$. It turns out that the $\mathrm{CH}$-invariants have a similar behaviour. Namely we show, in Corollary 2.8, that under the same assumptions one has that $p^{(k)}(b)=p^{(k)}\left(b^{\prime}\right)$.

X.-S. Lin showed in $[\mathbf{1 5}]$ that the $C H$-invariants are of finite type, in a certain sense. (At the same time, he also noticed that the $\mathrm{CH}$-invariants cannot be determined by quantum invariants.) Although less general than the finite type invariants, the $C H$-invariants have the following advantage, from the practical point of view. The theory of finite type invariants is a general scheme, that is, even if one knows that $\widehat{b}$ and $\widehat{b^{\prime}}$ can be distinguished by higher order Vassiliev invariants, it is in general hard to find a specific higher order invariant which does the job. The $\mathrm{CH}$-theory is more concrete, since it disposes of a built-in linear decision test for the equality at step $p^{(k+1)}$, whenever one has equality at step $p^{(k)}$; see [22, Theorem 1.4]. A similar non-linear decision test was introduced by Habegger-Lin in [9] to describe their solution of the link-homotopy classification problem.

Our main results in this paper are contained in Section 2. Lemmas 2.2-2.6 describe a complete simple set of rules for the Artin calculus of longitudes of pure braids, $\underline{l}(b)$, modulo the lower central series. A key tool is provided by a general result of independent interest, namely Proposition 2.1. It is wellknown how to associate a graded Lie algebra to a certain type of filtrations on a group (in particular, to the lower central series filtration); see [14]. For any given group $G$, one may consider the Torelli group, $T_{G}$, consisting of those automorphisms of $G$ which act trivially on the abelianization. The Torelli group is naturally endowed with the Torelli filtration, which gives rise to an associated graded Lie algebra. We establish, in Proposition 2.1, a faithful relationship between this graded Lie algebra and the graded Lie algebra of derivations of the graded Lie algebra associated to $\left\{\Gamma_{k} G\right\}$, for any group $G$. This is done via the Johnson homomorphism, introduced and exploited in [10], in connection with the study of the mapping class groups, in the case where $G$ is a surface group. The connection with link theory comes from the well-known fact that the Artin representation embeds $P_{n}$ into $T\left(\mathbf{F}_{X}\right)$; see e.g., (1.1). In Theorem 2.7 and Corollary 2.9 we show that 
the Torelli filtration coincides with the lower central series filtration of $P_{n}$ (which is not true in general; see [10]), both being detected either by the vanishing of the $C H$-invariants, or by the vanishing of $\underline{l}$ modulo the lower central series of $\mathbf{F}_{X}$.

The graded Lie algebra associated to the lower central series of $G$ will be denoted by $\operatorname{gr}_{\Gamma}^{*} G=: \oplus_{k \geq 1} \operatorname{gr}_{\Gamma}^{k} G$, where $\operatorname{gr}_{\Gamma}^{k} G=: \Gamma_{k} G / \Gamma_{k+1} G$. Note that $\operatorname{gr}_{\Gamma}^{*} P_{n}$ is torsion-free as a graded abelian group, and also that its graded Lie algebra structure is rather well-understood; see $[\mathbf{3}, \mathbf{7}, \mathbf{1 3}]$.

Let $b, b^{\prime} \in P_{n}$ be of the form $b=\beta \alpha$ and $b^{\prime}=\beta^{\prime} \alpha$, with $\alpha \in P_{n}$ and $\beta, \beta^{\prime} \in \Gamma_{k} P_{n}$. We present a first improvement of our general $C H$-test in Corollary 2.8. More precisely, we show that in this case $p^{(k)}(b)=p^{(k)}\left(b^{\prime}\right)$, and that $p^{(k+1)}(b)=p^{(k+1)}\left(b^{\prime}\right)$ if and only if the class of $\beta^{\prime} \beta^{-1}$ modulo $\Gamma_{k+1} P_{n}$, denoted by $\overline{\beta^{\prime} \beta^{-1}} \in \operatorname{gr}_{\Gamma}^{k} P_{n} \subset\left(\operatorname{gr}_{\Gamma}^{k} P_{n}\right) \otimes \mathbf{Q}$, belongs to a certain indeterminacy subspace of $\left(\operatorname{gr}_{\Gamma}^{k} P_{n}\right) \otimes \mathbf{Q}$, which depends on $\alpha$.

We take a second step in Section 3, where we introduce the homogenous links and pure braids. Here our main result is Theorem 3.2. It provides a major improvement of the general $p^{(k+1)}$-test, for the case of two pure braids, $b=\beta \alpha$ and $b^{\prime}=\beta^{\prime} \alpha$, as above, under a homogeneity assumption on $\alpha \in \Gamma_{h} P_{n}$, with $h<k$. Namely we give a description of the above indeterminacy subspace, which involves only the class of $\alpha$ modulo $\Gamma_{h+1} P_{n}$, $\bar{\alpha} \in \operatorname{gr}_{\Gamma}^{h} P_{n}$.

Proposition 3.3 offers two general homogeneity criteria. They are used to check the homogeneity of several classes of examples of pure braids, in Corollary 3.5, and to prove, in Corollary 3.6, the fact that all algebraic links (in the sense of $[\mathbf{2 0}, \S 10]$ ) are homogenous.

We illustrate in $\S 6$ the efficiency of our homogenous $\mathrm{CH}$-tests, on three classes of examples of pure links.

We exhibit, in Proposition 6.1, a series of infinite families, having the same Vassiliev invariants up to order $k-1$ and the same $\bar{\mu}$-invariants (of arbitrarily high order), which are faithfully detected by $p^{(k+1)}$, up to oriented (not necessarily ordered) isotopy. In Propositions 6.2 and 6.3 we present two infinite families of pairs of pure links, which are detected by $p^{(4)}$, up to oriented (unordered) isotopy. The examples from 6.2 are link-homotopic and have the same sublinks, while the examples from 6.3 are link-homotopic and share the same HOMFLY polynomial and the same $\bar{\mu}$-invariants (of arbitrarily high order).

\section{1. $C H$-invariants of pure braids.}

We start by recalling the construction of our $\mathrm{CH}$-invariants for the case of pure links, following [22]. A braid on $n$ strings $b \in B_{n}$ acts (on the right) on the free group on $n$ letters, $\mathbf{F}_{X}=: \mathbf{F}\left(x_{1}, \ldots, x_{n}\right)$, via the Artin representation. If $b$ is pure $\left(b \in P_{n}\right)$, one knows that this action is of the 
following form:

$$
x_{i} b={ }^{v_{i}} x_{i}, \quad \text { for } i=1, \ldots, n,
$$

with $v_{i} \in \mathbf{F}_{X}$ (where ${ }^{v} x=v x v^{-1}$ stands for the group conjugation); see [3]. If one normalizes by requiring that

$$
e_{i}\left(v_{i}\right)=0, \quad \text { for } i=1, \ldots, n,
$$

(where $e_{i}(v)$ equals the exponent sum of the letter $x_{i}$ in the free word $v$ ), one gets well-defined algebraic longitudes

$$
l_{i}(b)=v_{i}, \quad \text { for } \quad i=1, \ldots, n .
$$

Next one considers the Campbell-Hausdorff group embedding

$$
\rho: \mathbf{F}_{X} \longrightarrow \widehat{\mathbf{L}}_{X}
$$

defined on generators by $\rho\left(x_{i}\right)=x_{i}$, for $i=1, \ldots, n$. Here $\widehat{\mathbf{L}}_{X}$ is the free complete $\mathbf{Q}$-Lie algebra on $x_{1}, \ldots, x_{n}$. Its elements are formal Lie series in the indeterminates $x_{1}, \ldots, x_{n}$ with rational coefficients. It carries the group law provided by the well-known Campbell-Hausdorff formula; see $[\mathbf{1 6}, \mathbf{2 3}]$. Set then

$$
\rho l_{i}(b)=s_{i}=\sum_{r \geq 1} s_{i}^{r}, \quad \text { with } \quad s_{i}^{r} \in \mathbf{L}_{X}^{r}
$$

(where $\mathbf{L}_{X}^{r}$ denotes the $\mathbf{Q}$-span of those Lie monomials which are homogenous of bracket length $r$ ), for each $i=1, \ldots, n$.

One may further consider the free bigraded Lie algebra $\mathbf{L}_{*}^{*}=\mathbf{L}_{*}^{*}(X \oplus Y)$, freely generated by $X=\operatorname{span}_{\mathbf{Q}}\left\{x_{1}, \ldots, x_{n}\right\}, Y=\operatorname{span}_{\mathbf{Q}}\left\{y_{1}, \ldots, y_{n}\right\}$; the upper degree is given by bracket length and the lower degree comes from setting $|x|=0$ for $x \in X$ and $|y|=1$ for $y \in Y$. There is an associated bigraded Lie algebra of derivations, Der ${ }_{*}^{*}$, where $\operatorname{Der}_{j}^{r}$ stands for those Lie derivations of $\mathbf{L}_{*}^{*}$ which are bihomogeneous, of upper degree $+r(r \geq 0)$ and of lower degree $-j(j \geq 0)$. The Lie bracket is given by the (graded) commutator of derivations; see $[\mathbf{2 2}, \S 1]$. Everything in sight obeys to the standard Koszul sign convention with respect to the lower degrees; see e.g., [25].

One may associate to $b \in P_{n}$ the (formal) derivation $\partial(b)=\sum_{r \geq 1} \partial_{r}(b)$, with $\partial_{r}(b) \in \operatorname{Der}_{1}^{r}$ given for each $r \geq 1$ on the free generators by

$$
\begin{array}{ll}
\partial_{r}(b) x_{i}=0, & \text { for } \quad i=1, \ldots, n, \\
\partial_{r}(b) y_{i}=\left[x_{i}, s_{i}^{r}\right], & \text { for } i=1, \ldots, n,
\end{array}
$$

as in $[\mathbf{2 2},(2.1)-(2.2)$ and (1.3)].

Starting with two pure braids $b, b^{\prime} \in P_{n}$, the basic $C H$-test at step $k+1$ may be described as follows [22, Theorem 1.4]. Assume that

$$
s_{i}^{\prime r}=s_{i}^{r}, \quad \text { for every } \quad r<k \text { and } 1 \leq i \leq n,
$$


(where $\left.s_{i}^{\prime}=\rho l_{i}\left(b^{\prime}\right)\right)$. Then $p^{(k+1)}\left(b^{\prime}\right)=p^{(k+1)}(b)$ if and only if there exist $q_{t} \in \operatorname{Der}_{0}^{t}$, for $1 \leq t<k$, such that

$$
\sum_{r+t<k}\left[q_{t}, \partial_{r}(b)\right]=0
$$

and

$$
\sum_{r+t=k}\left[q_{t}, \partial_{r}(b)\right]=\partial_{k}(b)-\partial_{k}\left(b^{\prime}\right) .
$$

The main goal of this paper is to provide a much simpler form of the above test, for certain classes of pure braid examples, and then to illustrate the usefulness of this approach, by comparing it to various other known link invariants.

There is a general orbit space picture behind this, as explained in $[\mathbf{2 2}$, Introduction], see also [22, (1.3)-(1.4)]. If $K$ is an arbitrary $n$-component link (whose components are ordered and oriented), there are associated Milnor systems of algebraic longitudes [19] (see also [22, Theorem 1.1]). Such a system $\underline{l}$ provides for each $i(1 \leq i \leq n)$ a sequence $\left\{l_{i}^{(k)}\right\}_{k}$ of elements of $\mathbf{F}_{X}=\mathbf{F}\left(x_{1}, \ldots, x_{n}\right)$, which gives rise to $s_{i}=: \lim _{\longrightarrow} \rho\left(l_{i}^{(k)}\right)$. Here the limit is taken with respect to the topology of the natural complete filtration $\left\{F_{s} \widehat{\mathbf{L}}_{X}=: \widehat{\mathbf{L}}_{\bar{X}}^{\geq s}\right\}_{s}$ given by the order of the formal Lie series; see e.g., $[\mathbf{2 2}$, 2.2]. (In the case of a pure link $\widehat{b}$ one has $l_{i}^{(k)}=l_{i}(b)$, for all $k$.)

Denote by Der ${ }_{1}^{+}$the completion of $\oplus_{r \geq 1} \operatorname{Der}_{1}^{r}(\mathbf{L})$ with respect to the filtration $\left\{\oplus_{r \geq s} \operatorname{Der}_{1}^{r}\right\}_{s}$ and denote by $\left\{F_{s} \operatorname{Der}_{1}^{+}=: \prod_{r \geq s} \operatorname{Der}_{1}^{r}\right\}_{s}$ the induced filtration on $\operatorname{Der}_{1}^{+}$. Define $\partial(\underline{l}) \in \operatorname{Der}_{1}^{+}, \partial(\underline{l})=\sum_{r \geq 1} \partial_{r}(\underline{l})$, with $\partial_{r}(\underline{l}) \in \operatorname{Der}_{1}^{r}$, as in (1.6) above. One may also construct the free complete graded Lie algebra $\widehat{\mathbf{L}}_{*}$, as the completion of $\mathbf{L}$ with respect to the upper degree filtration; it is endowed with the induced order filtration, $\left\{F_{s} \widehat{\mathbf{L}}=\widehat{\mathbf{L}}^{\geq s}\right\}_{s}$, as before. It is then straightforward to reinterpret $\operatorname{Der}_{1}^{+}$as being the complete $\mathbf{Q}$ vector space of those Lie derivations of degree -1 of $\widehat{\mathbf{L}}_{*}$ which are strictly filtration-increasing. (See e.g., [22, Introduction and (1.3)].)

Denote by $U$ the pro-unipotent group of those filtration-preserving degree zero Lie automorphisms of $\widehat{\mathbf{L}}_{*}$ which act as the identity on the associated graded, as in [22, Introduction]. It is not hard to see that $U$ acts linearly by conjugation on the filtered vector space $\operatorname{Der}_{1}^{+}$. Denoting by $p^{(k)}(K)$ the $U$ orbit of $\partial(\underline{l})$ modulo $F_{k} \operatorname{Der}_{1}^{+}\left(p^{(k)}(K) \in U \backslash \operatorname{Der}_{1}^{+} / F_{k} \operatorname{Der}_{1}^{+}\right)$, we have shown in $[\mathbf{2 2}$, Theorem $\mathrm{A}]$ that $p^{(k)}(K)$ is independent of $\underline{l}$. It is an invariant of the (ordered and oriented) concordance class of $K$. Moreover (1.7)-(1.9) above correspond to the $C H$-test $p^{(k+1)}\left(\widehat{b^{\prime}}\right)=p^{(k+1)}(\widehat{b})$, under the assumption $p^{(k)}\left(\widehat{b^{\prime}}\right)=p^{(k)}(\widehat{b})$, see [22, Theorem 1.4]. 
By their construction, the $C H$-invariants are non-numerical invariants, lying in orbit spaces of (finite-dimensional) linear $U$-representations in $\operatorname{Der}_{1}^{+} / F_{k} \operatorname{Der}_{1}^{+}=\oplus_{r=1}^{k-1} \operatorname{Der}_{1}^{r}$, for $k \geq 2$. One knows [18] that for every $k$ this $U$-representation factors through $U_{k}$, the canonical unipotent subgroup of $G L\left(\operatorname{Der}_{1}^{+} / F_{k} \operatorname{Der}_{1}^{+}\right)$associated to the above direct sum decomposition. The failure of numerical invariant theory comes from the fact that the polynomial invariants of $U_{k}$ in $\operatorname{Der}_{1}^{+} / F_{k}$ reduce to the subalgebra of polynomials on $\operatorname{Der}_{1}^{1}$, for every $k$; thus they are quite inefficient for detecting $U_{k}$-orbits, for $k \geq 3$. At the same time, the invariant theory of the $U$-action on $\operatorname{Der}_{1}^{+} / F_{k}$ seems to be intractable.

What can be done instead is to use the linear decision test (1.8)-(1.9). We shall see its usefulness, on examples, in Section 6.

There is also a linear natural action of the semidirect product $\Phi=\mathbf{Z}_{2}^{n} \times \Sigma_{n}$ on $\operatorname{Der}_{1}^{+}$, which respects the filtration of $\operatorname{Der}_{1}^{+}$and normalizes the previous $U$-action; details may be found in the discussion preceding Proposition 2.8 [22]. It has the property that the $\Phi \cdot U$-orbit of $\partial(\underline{l})$ modulo $F_{k} \operatorname{Der}_{1}^{+}$, to be denoted by $\widetilde{p}^{(k)}(K)$, depends only on the unordered and unoriented concordance class of $K$; see [22, Proposition 2.8].

Remark 1.1. The $C H$-invariants are faithful at the basepoint, on pure links, that is, if $b \in P_{n}$ and $\widetilde{p}^{(k)}(b)=\widetilde{p}^{(k)}(1)$, for all $k$, then necessarily $b=1$.

Indeed, $l_{i}(1)=1$, for $i=1, \ldots, n$, whence $\partial(1)=0$. The equality of the $C H$-invariants forces then $\partial(b) \equiv 0$ modulo $F_{k} \operatorname{Der}_{1}^{+}$, for all $k$, whence $\left[x_{i}, s_{i}^{r}\right]=0$, for $i=1, \ldots, n$ and $r \geq 1$. One may use now the commutation properties of free Lie algebras [16, p. 328] (plus a normalization argument for $r=1$, see the beginning of the proof of Theorem $4.3[\mathbf{2 2}])$ to infer that $\rho\left(l_{i}(b)\right)=0$, for $i=1, \ldots, n$. The injectivity properties of the $C H$ representation and of the Artin representation imply then that $b=1$.

\section{Braid commutators and the Artin calculus of longitudes.}

We want to explain how to handle the $C H$-decision test (1.8)-(1.9) for pairs of pure braids of the form $b=\beta \alpha, b^{\prime}=\beta^{\prime} \alpha$, with $\alpha \in P_{n}$ and $\beta, \beta^{\prime} \in \Gamma_{k} P_{n}$. Here $\Gamma_{k} G$ denotes the $k$ th term of the lower central series of a group $G$ (defined by $\Gamma_{1} G=G$ and inductively $\Gamma_{k} G=\left(G, \Gamma_{k-1} G\right.$ ), where $(x, y)=x y x^{-1} y^{-1}$ stands for the commutator of two group elements). We have to begin by closely examinating the relationship between the lower central series of $P_{n}$ and of $\mathbf{F}_{X}$, via the construction of the algebraic longitudes ((1.1)-(1.3)). This may be done within the framework of the Johnson homomorphism. Our first result in this section generalizes a basic construction of Johnson [10], which turned out to be extremely useful in the study of mapping class groups, corresponding to the case where $G$ is a surface group. 
One may associate to any given group $G$ the following objects: The graded Lie algebra $\operatorname{gr}_{\Gamma}^{*} G=: \oplus_{k \geq 1} \Gamma_{k} G / \Gamma_{k+1} G$, with Lie bracket induced by the group commutator (there are no extra signs in the Lie identities!); the graded Lie algebra of derivations, $\operatorname{Der}^{*}\left(\operatorname{gr}_{\Gamma} G\right)=\oplus_{s \geq 1} \operatorname{Der}^{s}\left(\operatorname{gr}_{\Gamma} G\right)$ (where $\operatorname{Der}^{s}$ stands for the degree $s$ homogenous Lie derivations); the Torelli group $T_{G}$,

$$
T_{G}=:\left\{a \in \text { Aut } G \mid a \equiv i d \bmod \Gamma_{2} G\right\}
$$

and the decreasing filtration of $T_{G}, F_{s} T_{G}(s \geq 1)$,

$$
F_{s} T_{G}=:\left\{a \in \text { Aut } G \mid a \equiv i d \bmod \Gamma_{s+1} G\right\} .
$$

By an old result of Kaloujnine [12] one has $\left(F_{s}, F_{t}\right) \subset F_{s+t}$, for all $s, t \geq$ 1 , hence the commutator of automorphisms induces a graded Lie algebra structure on $\operatorname{gr}_{F}^{*} T_{G}=: \oplus_{s \geq 1} F_{s} T_{G} / F_{s+1} T_{G}$ and $\Gamma_{s} T_{G} \subset F_{s} T_{G}$, for all $s \geq 1$. We are ready to state our result:

Proposition 2.1. The Johnson construction which associates to a $\in T_{G}$ and $x \in G$ the element $a x \cdot x^{-1} \in G$ induces a graded Lie algebra monomorphism

$$
J: \operatorname{gr}_{F}^{*} T_{G} \longrightarrow \operatorname{Der}^{*}\left(\operatorname{gr}_{\Gamma} G\right)
$$

for any group $G$.

Proof. We are going to use the notation $(\cdot) \equiv_{k}(\cdot)$ to indicate either the equality of two elements of $T_{G}$ modulo $F_{k} T_{G}$ or the equality of two elements of $G$ modulo $\Gamma_{k} G$.

First we claim that $a x \equiv_{s+k} x$, for any $a \in F_{k} T_{G}$ and $x \in \Gamma_{s} G$. For $s=1$ this is just the definition of $F_{k}$, so we may proceed inductively. Pick up any $x \in \Gamma_{s} G$ and $y \in G$ and compute $a(x, y)=(a x, a y)=\left(x x_{s+k}, a y\right)$ (with $x_{s+k} \in \Gamma_{s+k}$, by induction) $\equiv_{s+k+1}(x, a y)$ (by standard commutator calculus, see e.g., $[\mathbf{1 6}])=\left(x, y y_{k+1}\right) \equiv_{s+k+1}(x, y)$ (by a similar argument), whence our claim. We thus have for each $a \in F_{k}$ and $s \geq 1$ a map $\widetilde{a}$ : $\Gamma_{s} \longrightarrow \Gamma_{s+k}$, defined by $\widetilde{a} x=a x \cdot x^{-1}$, inducing $\widetilde{a}: \Gamma_{s} \longrightarrow \operatorname{gr}_{\Gamma}^{s+k}$. If $x, y \in \Gamma_{s}$ then $a(x y) \cdot(x y)^{-1}=a x\left(a y \cdot y^{-1}\right) x^{-1} \equiv_{s+k+1}\left(a x \cdot x^{-1}\right)\left(a y \cdot y^{-1}\right)$ (by elementary commutator calculus). Therefore $\widetilde{a}$ induces an additive map $\widetilde{a}: \operatorname{gr}_{\Gamma}^{s} \longrightarrow \operatorname{gr}_{\Gamma}^{s+k}$. For any $a, b \in F_{k}$ and $x \in \Gamma_{s}$ we have $a(b x) \cdot x^{-1}=$ $a\left(b x \cdot x^{-1}\right) \cdot\left(a x \cdot x^{-1}\right) \equiv_{s+k+1}\left(b x \cdot x^{-1}\right)\left(a x \cdot x^{-1}\right)\left(\right.$ since $b x \cdot x^{-1} \in \Gamma_{s+k}$ and $\left.a \in F_{1}\right)$. Consequently setting $J(a)=\widetilde{a}$, for $a \in F_{k} T_{G}$, we obtain a well-defined additive degree zero map defined on $\operatorname{gr}_{F}^{*} T_{G}$ with values in the homogenous additive endomorphisms of positive degree of $\operatorname{gr}_{\Gamma}^{*} G$, which is injective by the very definition of the filtration of $T_{G}$.

To finish the proof, two more (less trivial) verifications are needed: The fact that $\widetilde{a}$ is a Lie derivation of $\operatorname{gr}_{\Gamma}^{*} G$, and the compatibility of $J$ with the Lie brackets.

Choose then arbitrarily $a \in F_{k}, x \in \Gamma_{s}$ and $y \in \Gamma_{t}$. We have to show that $\widetilde{a}(x, y) \equiv_{k+s+t+1}(\widetilde{a} x, y)(x, \widetilde{a} y)$. We begin by following the definitions 
and writing

$$
\widetilde{a}(x, y)=a x \cdot a y \cdot a x^{-1}\left(a y^{-1} \cdot y\right)\left(x, y^{-1}\right) y^{-1} .
$$

We may next commute $a y^{-1} \cdot y \in \Gamma_{k+t}$ with $\left(x, y^{-1}\right) \in \Gamma_{s+t}$, modulo $\Gamma_{k+s+t+1}$, expand $(\widetilde{a} x, y)(x, \widetilde{a} y)$ and simplify to get the equivalent congruence

$$
a y \cdot a x^{-1} \cdot x \equiv_{k+s+t+1} x^{-1} y x \cdot a x^{-1} \cdot y^{-1} x \cdot a y .
$$

This in turn may be rewritten as

$$
y\left(y^{-1} \cdot a y\right)\left(a x^{-1} \cdot x\right) \equiv_{k+s+t+1} y\left(y^{-1}, x^{-1}\right)\left(a x^{-1} \cdot x\right) x^{-1} y^{-1} x \cdot a y,
$$

in order to perform again a commutation trick (applied to both the above pairs of parentheses). Finally, after the simplification of both sides by $y\left(a x^{-1} \cdot x\right)$ and a little cancelation, this reduces to the identity $y^{-1} \cdot a y$ $\equiv_{k+s+t+1} y^{-1} \cdot a y$, so we are done.

To see that $J$ is a Lie algebra map we need to show that

$$
a b a^{-1} b^{-1}(x) \cdot x^{-1} \equiv_{k+s+t+1} \widetilde{a}\left(b x \cdot x^{-1}\right) \cdot\left[\widetilde{b}\left(a x \cdot x^{-1}\right)\right]^{-1},
$$

for any $a \in F_{s}, b \in F_{t}$ and $x \in \Gamma_{k}$. We may first rewrite $\widetilde{(a, b)} x$ as follows

$$
\begin{aligned}
& a b a^{-1} b^{-1}(x) \cdot x^{-1} \\
& \quad=a b\left\{\left[a^{-1}\left(b^{-1} x \cdot x^{-1}\right)\left(b^{-1} x \cdot x^{-1}\right)^{-1}\right] b^{-1} x \cdot x^{-1}\right\} a b a^{-1}(x) \cdot x^{-1} .
\end{aligned}
$$

Note that $b^{-1} x \cdot x^{-1} \in \Gamma_{k+t}$, therefore $\widetilde{a^{-1}}\left(b^{-1} x \cdot x^{-1}\right) \in \Gamma_{k+s+t}$. It follows that $\left.a b \widetilde{\left(a^{-1}\right.}\left(b^{-1} x \cdot x^{-1}\right)\right) \equiv_{k+s+t+1} \widetilde{a^{-1}}\left(b^{-1} x \cdot x^{-1}\right)$, since $a b \in F_{1}$. We may thus infer that

$$
\begin{aligned}
\widetilde{(a, b)} x \equiv_{k+s+t+1} \widetilde{a^{-1}}\left(b^{-1} x \cdot x^{-1}\right) & \\
& \cdot a b\left\{\left(b^{-1} x \cdot x^{-1}\right)\left(a^{-1} x \cdot x^{-1}\right)\right\} \cdot a b(x) \cdot x^{-1} .
\end{aligned}
$$

The already established linearity properties of $J$ together with the bilinearity of the commutator of derivations allow us to replace $a$ and $b$ by their inverses in the right-hand side of (2.1). Comparing the result with the above computation for $\widetilde{(a, b)} x$ we get the equivalent congruence

$$
\begin{aligned}
a b\left\{\left(b^{-1} x \cdot x^{-1}\right)\left(a^{-1} x \cdot x^{-1}\right)\right\} \cdot a b(x) \cdot x^{-1} & \\
& \equiv_{k+s+t+1} a^{-1} x \cdot x^{-1} \cdot b^{-1} x \cdot b^{-1} a^{-1}\left(x^{-1}\right) .
\end{aligned}
$$

It is convenient to write the left-hand side of $(2.2)$ in the form

$$
a\left(x \cdot b x^{-1}\right) \cdot a\left\{\widetilde{b}\left(a^{-1} x \cdot x^{-1}\right) \cdot\left(a^{-1} x \cdot x^{-1}\right)\right\} \cdot a\left(x \cdot b x^{-1}\right)^{-1} \cdot a x \cdot x^{-1} .
$$

Note that $x \cdot b x^{-1}, a\left(x \cdot b x^{-1}\right) \in \Gamma_{k+t}, a^{-1} x \cdot x^{-1}, a\left(a^{-1} x \cdot x^{-1}\right) \in \Gamma_{k+s}$ and consequently $\widetilde{b}\left(a^{-1} x \cdot x^{-1}\right), a \widetilde{b}\left(a^{-1} x \cdot x^{-1}\right) \in \Gamma_{k+s+t}$, which enables us 
to replace the above expression (using a commutation argument modulo $\Gamma_{k+s+t+1}$ ) by

$$
a b b^{-1}\left\{b\left(a^{-1} x \cdot x^{-1}\right) \cdot x \cdot a^{-1} x^{-1}\right\} .
$$

This equals

$a b\left(a^{-1} x \cdot x^{-1} \cdot b^{-1} x \cdot b^{-1} a^{-1}\left(x^{-1}\right)\right) \equiv_{k+s+t+1} a^{-1} x \cdot x^{-1} \cdot b^{-1} x \cdot b^{-1} a^{-1}\left(x^{-1}\right)$, as desired (see $(2.2))$, since $b^{-1} \widetilde{b}\left(a^{-1} x \cdot x^{-1}\right) \in \Gamma_{k+s+t}$ and $a b \in F_{1}$. Our proof is complete.

The next series of lemmas completely describes the calculus of longitudes modulo the lower central series of $P_{n}$ and of $\mathbf{F}_{X}$.

Lemma 2.2. If $\alpha \in \Gamma_{s} P_{n}$ then $l_{i}(\alpha) \in \Gamma_{s} \mathbf{F}_{X}$, for $i=1, \ldots, n$.

Proof. The abelianization of (1.1) readily implies that the Artin representation embeds $P_{n}$ into the Torelli group $T\left(\mathbf{F}_{X}\right)$, and consequently $\Gamma_{s} P_{n} \subset$ $F_{s} T\left(\mathbf{F}_{X}\right)$, for all $s \geq 1$. By the definition of the Torelli filtration this implies that $0 \equiv_{s+1} x_{i} \alpha \cdot x_{i}^{-1}=\left(l_{i}(\alpha), x_{i}\right)$ (see $(1.1)$ ), for $i=1, \ldots, n$. As is well-known $\operatorname{gr}_{\Gamma}^{*} \mathbf{F}_{X}$ is isomorphic to $\mathbf{L}_{X}^{*}(\mathbf{Z})$ (the free graded $\mathbf{Z}$-Lie algebra on the degree one generators $\left.x_{1}, \ldots, x_{n}\right)$. One also knows $([\mathbf{1 6}$, p. 328]) that the only homogenous elements of $\mathbf{L}_{X}^{*}(\mathbf{Z})$ which commute with $x_{i}$ are the multiples of $x_{i}$.

We may use these facts to show that $l_{i}(\alpha) \equiv_{t} 0$, for $t \leq s$, by induction. We need only to recall that $l_{i}(\alpha) \equiv_{2} \sum_{j \neq i} \mu_{i j} x_{j}$ (as a consequence of the normalization, see (1.2)), which completes the first nontrivial step $(t=$ $2)$.

Set then $\bar{l}_{i}(\alpha)=l_{i}(\alpha) \bmod \Gamma_{s+1} \mathbf{F}_{X}(i=1, \ldots, n)$, for $\alpha \in \Gamma_{s} P_{n}$.

Lemma 2.3. The above map induces a well-defined additive map $\bar{l}_{i}: \operatorname{gr}_{\Gamma}^{s} P_{n}$ $\longrightarrow \operatorname{gr}_{\Gamma}^{s} \mathbf{F}_{X}=\mathbf{L}_{X}^{s}(\mathbf{Z})$, for $i=1, \ldots, n$.

Proof. Apply the general Artin calculus rule

$$
l_{i}(\alpha \beta)=l_{i}(\alpha) \beta \cdot l_{i}(\beta)
$$

(see [22, Lemma 3.2]) to $\alpha, \beta \in \Gamma_{s} P_{n}$ and next to $\alpha \in \Gamma_{s} P_{n}, \beta \in \Gamma_{s+1} P_{n}$, together with the preceding result.

The next key lemma appeals to the delicate part of Proposition 2.1 (the compatibility of $J$ with the Lie algebra structures).

Lemma 2.4. Defining, for $\gamma \in \Gamma_{k} P_{n}, \widetilde{\gamma} \in \operatorname{Der}^{k} \mathbf{L}_{X}(\mathbf{Z})$ by $\widetilde{\gamma} x_{i}=\left[x_{i}, \bar{l}_{i}(\gamma)\right]$, for $i=1, \ldots, n$ (on the free Lie algebra generators) one has for any $\alpha \in$ $\Gamma_{s} P_{n}$ and $\beta \in \Gamma_{t} P_{n}$

$$
\bar{l}_{i}(\alpha, \beta)=\left[\bar{l}_{i}(\alpha), \bar{l}_{i}(\beta)\right]+\widetilde{\alpha}\left(\bar{l}_{i}(\beta)\right)-\widetilde{\beta}\left(\bar{l}_{i}(\alpha)\right), \quad \text { for } \quad i=1, \ldots, n .
$$


Proof. Let $\lambda: \operatorname{gr}_{\Gamma}^{*} P_{n} \longrightarrow \operatorname{Der}^{*}\left(\mathbf{L}_{X}(\mathbf{Z})\right)$ be the negative of the composition of the map induced by the Artin representation, $\operatorname{gr}_{\Gamma}^{*} P_{n} \longrightarrow \operatorname{gr}_{F}^{*} T\left(\mathbf{F}_{X}\right)$, followed by Johnson's homomorphism $\operatorname{gr}_{F}^{*} T\left(\mathbf{F}_{X}\right) \longrightarrow \operatorname{Der}^{*}\left(\mathbf{L}_{X}(\mathbf{Z})\right)$. We know from Proposition 2.1 that $\lambda$ is a graded Lie algebra map (the minus sign comes from the fact that $P_{n}$ acts on $\mathbf{F}_{X}$ on the right via Artin's representation). By construction we have for any $\gamma \in \Gamma_{k} P_{n}$

$$
\lambda(\gamma) x_{i} \equiv_{k+2}\left(x_{i} \gamma \cdot x_{i}^{-1}\right)^{-1},
$$

which may be rewritten (see (1.1)) in additive notation as $\left[x_{i}, \bar{l}_{i}(\gamma)\right]$, i.e., $\lambda(\gamma)=\widetilde{\gamma}$. The fact that $\lambda$ is a Lie map implies that

$$
\begin{aligned}
{\left[x_{i}, \bar{l}_{i}(\alpha, \beta)\right] } & =\lambda(\alpha, \beta) x_{i}=\widetilde{\alpha}\left(\widetilde{\beta} x_{i}\right)-\widetilde{\beta}\left(\widetilde{\alpha} x_{i}\right) \\
& =\widetilde{\alpha}\left[x_{i}, \bar{l}_{i}(\beta)\right]-\widetilde{\beta}\left[x_{i}, \bar{l}_{i}(\alpha)\right] \\
& =\left[x_{i}, \widetilde{\alpha}\left(\bar{l}_{i} \beta\right)-\widetilde{\beta}\left(\bar{l}_{i} \alpha\right)\right]+\left[\left[x_{i}, \bar{l}_{i} \alpha\right], \bar{l}_{i} \beta\right]-\left[\left[x_{i}, \bar{l}_{i} \beta\right], \bar{l}_{i} \alpha\right]
\end{aligned}
$$

(since $\widetilde{\alpha}$ and $\widetilde{\beta}$ are Lie derivations)

$$
=\left[x_{i},\left[\bar{l}_{i} \alpha, \bar{l}_{i} \beta\right]+\widetilde{\alpha}\left(\bar{l}_{i} \beta\right)-\widetilde{\beta}\left(\bar{l}_{i} \alpha\right)\right]
$$

(by Jacobi). Invoking once more the commutation properties of a free Lie algebra [16, p. 328], we get our stated formula.

The previous lemmas offer a complete simple recipe of computation of the algebraic longitudes modulo the lower central series, as soon as one knows the values of $\bar{l}_{i}(1 \leq i \leq n)$ on a specific set of group generators of $P_{n}$. We are going to use the well-known generators $\left(a_{r s}\right)_{1 \leq r<s \leq n}$, see $[\mathbf{3}, 1.8 .2]$.

Lemma 2.5. The values of $\bar{l}_{i}\left(a_{r s}\right) \in \mathbf{L}_{X}^{1}(\mathbf{Z})=\operatorname{gr}_{\Gamma}^{1} \mathbf{F}_{X}$, for $1 \leq r<s \leq n$ and $1 \leq i \leq n$, are zero excepting $\bar{l}_{r}\left(a_{r s}\right)=x_{s}$ and $\bar{l}_{s}\left(a_{r s}\right)=x_{r}$.

Proof. The precise form of $(1.1)$ for $b=a_{r s}$ is given in [3, 1.8.3]. It is a routine exercise to apply the definitions (1.2)-(1.3) and then to get the desired result by abelianization.

Here is one more useful result in the Artin calculus modulo commutators, involving the action of the symmetric group $\Sigma_{n}$, which comes from the conjugation in the full braid group $B_{n}$. (This corresponds to the passage from ordered oriented to unordered oriented isotopy type, via the Artin closure.) Firstly one has the natural action of $\Sigma_{n}$ on $\mathbf{L}_{X}^{*}(\mathbf{Z})$ by (degree zero) graded Lie algebra automorphisms which is given on the free generators by $g x_{i}=x_{g i}\left(g \in \Sigma_{n}, i \in\{1, \ldots, n\}\right)$. On the other hand there is a well-known short exact sequence of groups (see e.g., [3, p. 19])

$$
1 \longrightarrow P_{n} \longrightarrow B_{n} \stackrel{\nu}{\longrightarrow} \Sigma_{n} \longrightarrow 1 \text {. }
$$


Thus $B_{n}$ acts by conjugation on $\operatorname{gr}_{\Gamma}^{*} P_{n}$, by graded Lie algebra automorphisms, and since obviously the action of $P_{n}$ is trivial one has in fact an action of $\Sigma_{n}$, via $\nu$.

Denoting by $\overline{(\cdot)}$ the class modulo $\Gamma_{s+1}$ of an element belonging to $\Gamma_{s}$, we thus have by definition (for $g \in \Sigma_{n}$ and $\left.\alpha \in \Gamma_{s} P_{n}\right) g(\bar{\alpha})=\overline{\sigma_{\alpha}}$, where $\nu(\sigma)=g^{-1}$.

Lemma 2.6. For $g \in \Sigma_{n}, \alpha \in \Gamma_{s} P_{n}$ and $1 \leq i \leq n$ one has

$$
\bar{l}_{i}(g \bar{\alpha})=g \bar{l}_{g^{-1} i}(\bar{\alpha}) \text {. }
$$

Proof. Pick $\sigma \in B_{n}$ such that $\nu(\sigma)=g^{-1}$. One knows [3] that the Artin action of $\sigma$ on $\mathbf{F}_{X}$ is of the following form

$$
x_{i} \sigma={ }^{w_{i}} x_{g^{-1} i}, \quad \text { for } i=1, \ldots, n .
$$

According to [22, Lemma 3.5] one then has for every $i$

$$
l_{i}\left({ }^{\sigma} \alpha\right)=\left(w_{i} \alpha \cdot l_{g^{-1} i}(\alpha) \cdot w_{i}^{-1}\right) \sigma^{-1},
$$

where $w_{i} \in \mathbf{F}_{X}$ is as in (2.4). Using Lemma 2.2 we may compute modulo $\Gamma_{s+1}$ as follows

$$
l_{i}\left({ }^{\sigma} \alpha\right) \equiv_{s+1} l_{g^{-1} i}(\alpha) \sigma^{-1} \cdot\left(w_{i} \alpha \cdot w_{i}^{-1}\right) \sigma^{-1} \equiv_{s+1} l_{g^{-1} i}(\alpha) \sigma^{-1}
$$

(since $\alpha \in \Gamma_{s} P_{n} \subset F_{s} T\left(\mathbf{F}_{X}\right)$, whence $w \alpha \equiv_{s+1} w$, for any $w \in \mathbf{F}_{X}$ ). Finally we get $l_{g^{-1}}(\alpha) \sigma^{-1} \equiv_{s+1} g \bar{l}_{g^{-1} i}(\bar{\alpha})$, as desired, by noting that $l_{g^{-1} i}(\alpha) \in$ $\Gamma_{s} \mathbf{F}_{X}$ and remarking that the action of $\sigma^{-1}$ on $\Gamma_{s} \mathbf{F}_{X} / \Gamma_{s+1} \mathbf{F}_{X}=\operatorname{gr}_{\Gamma}^{s} \mathbf{F}_{X}$ is the Lie multiplicative extension of the action of $\sigma^{-1}$ on $\operatorname{gr}_{\Gamma}^{1} \mathbf{F}_{X}=\mathbf{L}_{X}^{1}(\mathbf{Z})$. This in turn may be easily read off from (2.4) by abelianization

$$
x_{j} \sigma^{-1} \equiv_{2} x_{g j}, \quad \text { for } j=1, \ldots, n,
$$

which finishes the proof.

The faithful relationship between the lower central series of $P_{n}$ and of $\mathbf{F}_{X}$ is described by the following more precise form of Lemma 2.2. It is also remarkable that in the case of $P_{n}$ the lower central series filtration coincides with the Torelli filtration, compare to $[\mathbf{1 0}, \S 7]$.

Theorem 2.7. If $\alpha \in P_{n}$ then the following are equivalent:

(i) $\alpha \in \Gamma_{s} P_{n}$.

(ii) $l_{i}(\alpha) \in \Gamma_{s} \mathbf{F}_{X}$, for every $i=1, \ldots, n$.

(iii) $\alpha \in F_{s} T\left(\mathbf{F}_{X}\right)$.

Proof. (i) $\Leftrightarrow$ (ii). Plainly it will be enough to assume inductively that $\alpha \in \Gamma_{k} P_{n}$ (for some $k \geq 1$ ) and prove that $\alpha \in \Gamma_{k+1} P_{n}$, if $\bar{l}_{i}(\alpha)=0$ for every $i$. This assertion in turn (which is trivial for $n=1$ ) will be proved by induction on $n$. It will follow for $P_{n}$ by making use of the basic split exact sequence $[3$, p. 23]

$$
1 \longrightarrow K_{n} \longrightarrow P_{n} \stackrel{\eta}{\longrightarrow} P_{n-1} \longrightarrow 1 \text {. }
$$


In terms of Artin representations, if $\alpha \in P_{n}$ is represented by

$$
\alpha: \mathbf{F}\left(x_{1}, \ldots, x_{n}\right) \stackrel{\sim}{\longrightarrow} \mathbf{F}\left(x_{1}, \ldots, x_{n}\right)
$$

then $\eta(\alpha)$ is represented by

$$
\alpha \bmod x_{n}: \mathbf{F}\left(x_{1}, \ldots, x_{n-1}\right) \stackrel{\sim}{\longrightarrow} \mathbf{F}\left(x_{1}, \ldots, x_{n-1}\right) .
$$

To construct the splitting, extend the action of $\beta \in P_{n-1}$ to $\mathbf{F}\left(x_{1}, \ldots, x_{n}\right)$, by setting

$$
x_{n} \beta=x_{n} .
$$

The split exact sequence with trivial $H_{1}$-monodromy (2.5) remains split exact after applying the functor $\Gamma_{k}$, as shown in [7]. Therefore $\alpha=u \beta$, with $u \in \Gamma_{k} K_{n}$ and $\beta \in \Gamma_{k} P_{n-1}$. Then the hypothesis $\bar{l}_{n}(\alpha)=0$ implies $\bar{l}_{n}(u)=0$ (by additivity, see Lemma 2.3, given that $l_{n}(\beta)=1$ by construction, see $(2.6))$.

On the other hand the formula [22, Lemma 3.2] $l_{n}(v \omega)=l_{n}(v) \omega \cdot l_{n}(\omega)$, taken modulo $x_{n}$ and applied to $v, \omega \in K_{n}$, which is the free group generated by $\left\{a_{r n} \mid 1 \leq r<n\right\}$ (see [3, p. 23]), identifies $l_{n} \bmod x_{n}: K_{n} \longrightarrow$ $\mathbf{F}\left(x_{1}, \ldots, x_{n-1}\right)$ with the group isomorphism sending $a_{r n}$ into $x_{r}$, for all $r<n$, see [3, 1.8.3]; compare also with [9, p. 400]. We may thus infer that $u \in \Gamma_{k+1} K_{n}$. The other hypotheses made on $\alpha$ imply that $\bar{l}_{i}(\beta)=0$ for $1 \leq i<n$, whence $\beta \in \Gamma_{k+1} P_{n-1}$ by induction and thus $\alpha=u \beta \in \Gamma_{k+1} P_{n}$.

(i) $\Leftrightarrow$ (iii). This time it will be enough to assume inductively that $\alpha \in$ $\Gamma_{k} P_{n} \cap F_{k+1} T\left(\mathbf{F}_{X}\right)$ and to infer that necessarily $\alpha \in \Gamma_{k+1} P_{n}$. Lemma 2.2 and the construction of $J$ (Proposition 2.1) imply that $\left[\bar{l}_{i}(\alpha), x_{i}\right]=0$ for $i=1, \ldots, n$. It follows, as in Lemma 2.2, that $l_{i}(\alpha) \in \Gamma_{k+1} \mathbf{F}_{X}$, for all $i$. The preceding result enables us to infer that $\alpha \in \Gamma_{k+1} P_{n}$, as needed.

Going back to the setting of the previous section, let $b, b^{\prime} \in P_{n}$ be of the form $b=\beta \alpha$ and $b^{\prime}=\beta^{\prime} \alpha$, with $\alpha \in P_{n}$ and $\beta, \beta^{\prime} \in \Gamma_{k} P_{n}$.

Define an additive map $\Lambda: \operatorname{gr}_{\Gamma}^{k} P_{n} \longrightarrow \operatorname{Der}_{1}^{k} \mathbf{L}(X \oplus Y)$ by $\Lambda(\bar{\gamma}) x_{i}=0$, $i=1, \ldots, n$ and

$$
\Lambda(\bar{\gamma}) y_{i}=\left[x_{i}, \bar{l}_{i}(\bar{\gamma})\right], \quad \text { for } \quad i=1, \ldots, n,
$$

where $\gamma \in \Gamma_{k} P_{n}$.

As a first corollary of our analysis of the Artin calculus modulo commutators we may offer the following result.

Corollary 2.8. If $b, b^{\prime} \in P_{n}$ are as above then $p^{(k)}\left(b^{\prime}\right)=p^{(k)}(b)$. More precisely:

(i) $s_{i}^{\prime r}=s_{i}^{r}$, for all $r<k$ and $1 \leq i \leq n$, as in (1.7).

(ii) $\partial_{r}\left(b^{\prime}\right)=\partial_{r}(b)=\partial_{r}(\alpha)$, for all $r<k$.

(iii) $\partial_{k}(b)-\partial_{k}\left(b^{\prime}\right)=\Lambda\left(\bar{\beta}-\overline{\beta^{\prime}}\right)$. 
Proof. The assertion (ii) follows from (i) (see (1.6)), which in turn may be proved as follows. One of the basic properties of the $\mathrm{CH}$-representation (1.4) is that if $v \equiv_{k} v^{\prime}\left(v, v^{\prime} \in \mathbf{F}_{X}\right)$ then the formal Lie series $\rho v$ and $\rho v^{\prime}$ are equal modulo terms of bracket length at least $k$; see $[\mathbf{1 4}, \mathbf{1 6}]$. With this remark, given the definition (1.5) of $s_{i}$ and $s_{i}^{\prime}$, the assertion to be proved is a consequence of the multiplicative property [22, Lemma 3.2] which says that

$$
l_{i}(b)=l_{i}(\beta) \alpha \cdot l_{i}(\alpha), \quad \text { for } \quad i=1, \ldots, n
$$

(and likewise for $\left.l_{i}\left(b^{\prime}\right)\right)$. Therefore $l_{i}(b) \equiv_{k} l_{i}(\alpha) \equiv_{k} l_{i}\left(b^{\prime}\right)$, since $\beta, \beta^{\prime} \in \Gamma_{k} P_{n}$ (use Lemma 2.2).

As for the last assertion, in order to compute $\partial_{k}(b)$ we will need $\rho l_{i}(b)$ modulo terms of bracket length at least $k+1$, for all $i$ (and similarly for $\left.\partial_{k}\left(b^{\prime}\right)\right)$. By the same argument as before we may reduce modulo $\Gamma_{k+1}$ in (2.8) and replace $l_{i}(b)$ by $l_{i}(\beta) \cdot l_{i}(\alpha)$ and $l_{i}\left(b^{\prime}\right)$ by $l_{i}\left(\beta^{\prime}\right) \cdot l_{i}(\alpha)$, since $l_{i}(\beta), l_{i}\left(\beta^{\prime}\right) \in$ $\Gamma_{k} \mathbf{F}_{X}$ and $\alpha \in T\left(\mathbf{F}_{X}\right)$. We recall now the $C H$-multiplication formula [16]

$$
s \cdot t=s+t+\frac{1}{2}[s, t]+\text { terms of higher bracket length }
$$

(where $s, t \in \widehat{\mathbf{L}}_{X}$ ). As we have already remarked, $l_{i}(\beta) \equiv_{k} 1$ implies that $\rho l_{i}(\beta)$ contains only terms of bracket length at least $k$. Therefore the above $C H$-multiplication formula reduces to $\rho l_{i}(\beta) \cdot \rho l_{i}(\alpha) \equiv \rho l_{i}(\beta)+\rho l_{i}(\alpha)$ modulo terms of degree at least $k+1$ (and likewise for $\rho l_{i}\left(\beta^{\prime}\right) \cdot \rho l_{i}(\alpha)$ ).

We infer that $\partial_{k}(b)=\partial_{k}(\beta)+\partial_{k}(\alpha)$ and $\partial_{k}\left(b^{\prime}\right)=\partial_{k}\left(\beta^{\prime}\right)+\partial_{k}(\alpha)$, by construction, whence $\partial_{k}(b)-\partial_{k}\left(b^{\prime}\right)=\partial_{k}(\beta)-\partial_{k}\left(\beta^{\prime}\right)$. Thus it will suffice to show that $\beta \in \Gamma_{k} P_{n}$ implies that $\partial_{k}(\beta)=\Lambda(\bar{\beta})$, in order to finish our proof. On the other hand this claim is a direct consequence of the definitions of $\partial_{k}$ and $\Lambda$, due to another basic property of the $C H$-representation [16], namely that $\rho v \equiv \bar{v}$ modulo terms of degree at least $k+1$, for any $v \in \Gamma_{k} \mathbf{F}_{X}$. Here $\bar{v}$ denotes as usual the class of $v$ modulo $\Gamma_{k+1} \mathbf{F}_{X}$, belonging to $\operatorname{gr}{ }_{\Gamma}^{k} \mathbf{F}_{X}=$ $\mathbf{L}_{X}^{k}(\mathbf{Z})$.

Our second corollary says that the lower central series filtration of pure braids may be detected by (unordered, unoriented) $\mathrm{CH}$-invariants; see also Remark 1.1.

Corollary 2.9. If $\alpha \in P_{n}$ then $\alpha \in \Gamma_{k} P_{n}$ if and only if $\widetilde{p}^{(k)}(\alpha)=0$, where $0 \in\left(\Phi \cdot U \backslash \operatorname{Der}_{1}^{+} / F_{k} \operatorname{Der}_{1}^{+}\right)$.

Proof. Since the action of $\Phi \cdot U$ on $\operatorname{Der}_{1}^{+}$is linear, the equality $\widetilde{p}^{(k)}(\alpha)=$ 0 translates to $\partial(\alpha) \in F_{k} \operatorname{Der}_{1}^{+}$. As in Remark 1.1, this is equivalent to $\rho l_{i}(\alpha) \equiv 0$ modulo $F_{k} \widehat{\mathbf{L}}_{X}$, for all $i$. Since $\rho$ is faithful with respect to the natural filtrations (see $[\mathbf{1 4}, \mathbf{1 6}]$ ) this in turn is equivalent to $l_{i}(\alpha) \in \Gamma_{k} \mathbf{F}_{X}$, for all $i$. Finally this means precisely that $\alpha \in \Gamma_{k} P_{n}$, by Theorem 2.7. 


\section{Homogenous links.}

Let $K$ be an arbitrary $n$-component link, ordered and oriented. As recalled in $\S 1$, the construction of the $C H$-invariants of $K$ starts with the choice of an algebraic system of longitudes, $\underline{l}=\left\{l_{i}^{(k)}\right\}$. One knows that $l_{i}^{(k+1)} \equiv l_{i}^{(k)}$ modulo $\Gamma_{k} \mathbf{F}_{X}$, therefore $\rho l_{i}^{(k+1)} \equiv \rho l_{i}^{(k)}$ modulo $\widehat{\mathbf{L}}_{\bar{X}}^{\geq k}$ and thus we get for each $i$ a formal Lie series $s_{i}=: \lim _{k} \rho l_{i}^{(k)} \in \widehat{\mathbf{L}}_{X}$. There is an associated Lie derivation of $\widehat{\mathbf{L}}_{*}, \partial=\partial(\underline{l}) \in \operatorname{Der}_{1}^{+}$, defined on the free Lie generators by $\partial x_{i}=0$ and $\partial y_{i}=\left[x_{i}, s_{i}\right]$, for $i=1, \ldots, n$.

Definition 3.1. A derivation $\partial \in \operatorname{Der}_{1}^{+}$of the form $\partial=\sum_{j \geq h} \partial_{j}$, where $\partial_{j} \in \operatorname{Der}_{1}^{j}$, for every $j$, is said to be $h$-homogenous if its $U$-orbit contains $\partial_{h}$. A link $K$ is $h$-homogenous if it has an algebraic system of longitudes $\underline{l}$ such that $\partial(\underline{l})$ is $h$-homogenous. A braid $\alpha \in P_{n}$ is $h$-homogenous if $\partial(\alpha)$ is $h$-homogenous.

Consider then $b=\beta \alpha$ and $b^{\prime}=\beta^{\prime} \alpha$, with $\alpha \in \Gamma_{h} P_{n}$ and $\beta, \beta^{\prime} \in \Gamma_{k} P_{n}$, $k>h \geq 1$. The following result provides a major simplification of the CH-test (1.8)-(1.9).

Theorem 3.2. If $b, b^{\prime} \in P_{n}$ are as above then:

(i) Their CH-invariants of order $k$ are equal, $p^{(k)}\left(b^{\prime}\right)=p^{(k)}(b)$.

(ii) If $\alpha$ is h-homogenous then $p^{(k+1)}\left(b^{\prime}\right)=p^{(k+1)}(b)$ if and only if the derivation $\Lambda\left(\bar{\beta}-\overline{\beta^{\prime}}\right)$ constructed as in (2.7) belongs to

$$
\operatorname{Im}\left\{\operatorname{ad}\left(\partial_{h}(\alpha)\right): \operatorname{Der}_{0}^{k-h} \mathbf{L}(X \oplus Y) \longrightarrow \operatorname{Der}_{1}^{k} \mathbf{L}(X \oplus Y)\right\},
$$

or equivalently if and only if there exists $\theta \in \operatorname{Der}^{k-h} \mathbf{L}_{X}^{*}$ with the property that for each $i=1, \ldots, n$ one has

$$
\begin{gathered}
{\left[x_{i}, \bar{l}_{i}\left(\bar{\beta}-\bar{\beta}^{\prime}\right)\right] \equiv\left[x_{i}, \theta \bar{l}_{i}(\bar{\alpha})\right]+\left[\theta x_{i}, \bar{l}_{i}(\bar{\alpha})\right]} \\
\text { modulo }\left\langle\left[x_{j}, \bar{l}_{j}(\bar{\alpha})\right] \mid 1 \leq j \leq n\right\rangle .
\end{gathered}
$$

Here $\bar{\beta}, \overline{\beta^{\prime}} \in \operatorname{gr}{ }_{\Gamma}^{k} P_{n}$ represent the classes of $\beta, \beta^{\prime}$ modulo $\Gamma_{k+1} P_{n}$ and likewise $\bar{\alpha} \in \operatorname{gr}_{\Gamma}^{h} P_{n}$ stands for the class of $\alpha$ modulo $\Gamma_{h+1} P_{n}$, and $\langle(\cdot)\rangle$ denotes the (homogenous) Lie ideal of $\mathbf{L}_{X}^{*}$ generated by $(\cdot)$.

Proof. The above statement (i) follows at once from Corollary 2.8.

For the proof of (ii) we will need the following general remarks. Denote by Der ${ }_{0}^{+}$the completion of $\oplus_{r \geq 1} \operatorname{Der}_{0}^{r}(\mathbf{L})$ with respect to the upper degree filtration. Note that we may identify $\mathrm{Der}_{0}^{+}$with the vector space of those degree zero Lie derivations of $\widehat{\mathbf{L}}_{*}$ which are strictly filtration-increasing, similarly as we did before in the case of $\operatorname{Der}_{1}^{+}$(see $\left.\S 1\right)$. As in that case, there is a natural action of $U$ on $\operatorname{Der}_{0}^{+}$, by conjugation. Moreover the Lie bracket of Der* ${ }_{*}^{*}$ induces (by linearity and completion) a bilinear map $\operatorname{Der}_{0}^{+} \times \operatorname{Der}_{1}^{+} \stackrel{[\cdot, \cdot]}{\longrightarrow} \operatorname{Der}_{1}^{+}$, 
which is obviously $U$-equivariant. Finally note that $\operatorname{gr}_{F}^{*}(u)=i d$, for any $u \in U$, where $\left\{F_{k} \operatorname{Der}_{1}^{+}\right\}_{k}$ is the canonical filtration of $\operatorname{Der}_{1}^{+}$; see $[\mathbf{1 8}, 1.8]$.

These remarks may be used to rephrase (1.8)-(1.9) as follows: $p^{(k+1)}\left(b^{\prime}\right)=$ $p^{(k+1)}(b)$ if and only if there exists $q \in \operatorname{Der}_{0}^{+}$with the properties

$$
[q, \partial(\alpha)] \equiv_{k} 0
$$

and

$$
[q, \partial(\alpha)] \equiv_{k+1} \partial(b)-\partial\left(b^{\prime}\right)
$$

Here we have used $\equiv_{s}$ to denote the equality of elements in Der ${ }_{1}^{+}$modulo $F_{s}$ Der $_{1}^{+}$and we have used Corollary 2.8 (ii) to replace $\partial_{r}(b)$ in (1.8)-(1.9) by $\partial_{r}(\alpha)$, for $r<k$.

If $\alpha$ is $h$-homogenous then by Definition 3.1 there exists $u \in U$ such that ${ }^{u} \partial(\alpha)=\partial_{h}(\alpha)$. Since the conjugation by $u$ is a filtered automorphism of Der $_{1}^{+}$and it is also compatible with the commutator of derivations, we may replace (3.1)-(3.2) by the equivalent condition: There exists $q \in \mathrm{Der}_{0}^{+}$such that

$$
\left[q, \partial_{h}(\alpha)\right] \equiv_{k} 0
$$

and

$$
\left[q, \partial_{h}(\alpha)\right] \equiv_{k+1}^{u}\left(\partial(b)-\partial\left(b^{\prime}\right)\right) \equiv_{k+1} \partial(b)-\partial\left(b^{\prime}\right) .
$$

(The second congruence from (3.4) comes from the fact that $\partial(b)-\partial\left(b^{\prime}\right) \equiv_{k} 0$, see Corollary 2.8 (ii), because the conjugation by $u$ is unipotent with respect to the filtration $\left\{F_{s} \mathrm{Der}_{1}^{+}\right\}$, as recalled before.)

Writing now $q=\sum_{j \geq 1} q_{j}$, with $q_{j} \in \operatorname{Der}_{0}^{j}(\mathbf{L})$, we may use the homogeneity of $\partial_{h}(\alpha)$ to rewrite (3.3)-(3.4) as follows

$$
\left[q_{j}, \partial_{h}(\alpha)\right]=0, \text { for } j<k-h
$$

and

$$
\left[q_{k-h}, \partial_{h}(\alpha)\right]=\partial_{k}(b)-\partial_{k}\left(b^{\prime}\right) .
$$

Summing up and resorting to Corollary 2.8 (iii) we find that $p^{(k+1)}\left(b^{\prime}\right)=$ $p^{(k+1)}(b)$ if and only if $\Lambda\left(\bar{\beta}-\overline{\beta^{\prime}}\right) \in \operatorname{ad}\left(\partial_{h}(\alpha)\right) \operatorname{Der}_{0}^{k-h} \mathbf{L}(X \oplus Y)$, as asserted. To get the stated equivalent form of this condition, reformulate (3.6) by applying both sides to $y_{i}, 1 \leq i \leq n$, to obtain by the definition of $\Lambda$ the equivalent form

$$
\left[x_{i}, \bar{l}_{i}\left(\bar{\beta}-\overline{\beta^{\prime}}\right)\right]=q_{k-h} \partial_{h}(\alpha) y_{i}-\partial_{h}(\alpha) q_{k-h} y_{i}, \text { for all } i .
$$

(Note that the derivations are uniquely determined by their values on the free generators and $\partial x_{i}=0$ for all $i$, if $\partial \in \operatorname{Der}_{1} \mathbf{L}(X \oplus Y)$, for trivial degree reasons.)

Remember next that $\alpha \in \Gamma_{h} P_{n}$, therefore $\partial_{h}(\alpha) y_{i}=\left[x_{i}, \bar{l}_{i}(\bar{\alpha})\right]$, for all $i$; see the proof of Corollary 2.8 (iii). Consequently $q_{k-h} \partial_{h}(\alpha) y_{i}=\left[x_{i}, \theta \bar{l}_{i}(\bar{\alpha})\right]+$ 
$\left[\theta x_{i}, \bar{l}_{i}(\bar{\alpha})\right]$, where $\theta \in \operatorname{Der}^{k-h} \mathbf{L}_{X}$ is given by the restriction of $q_{k-h}$ to $\mathbf{L}_{X}^{*}$. In fact a straightforward degree inspection shows $q_{k-h}$ to be uniquely determined on $X$ by $\theta \in \operatorname{Der}^{k-h} \mathbf{L}_{X}$ and on $Y$ by $q_{k-h} y_{i}=\sum_{j=1}^{n} c_{i j}$, with $c_{i j}$ $(1 \leq i, j \leq n)$ arbitrary elements belonging to $\operatorname{ad}_{X}^{k-h}\left(y_{j}\right)$. Here $\operatorname{ad}_{X}^{k-h}\left(y_{j}\right)$ is the Q-span of the Lie monomials of the form ad $\left(x_{i_{1}}\right) \cdots \operatorname{ad}\left(x_{i_{k-h}}\right)\left(y_{j}\right)$. We may thus see that $\left\{-\partial_{h}(\alpha) q_{k-h} y_{i}\right\}_{1 \leq i \leq n}$ is an arbitrary collection of $n$ elements of degree $k+1$ belonging to $\left\langle\left[x_{j}, \bar{l}_{j}(\bar{\alpha})\right] \mid 1 \leq j \leq n\right\rangle$, since $\partial \operatorname{ad}\left(x_{i_{1}}\right) \cdots \operatorname{ad}\left(x_{i_{s}}\right)\left(y_{j}\right)=\operatorname{ad}\left(x_{i_{1}}\right) \cdots \operatorname{ad}\left(x_{i_{s}}\right)\left(\partial y_{j}\right)$, for any $\partial \in \operatorname{Der}_{1} \mathbf{L}(X \oplus$ $Y)$ and $1 \leq i_{1}, \ldots, i_{s}, j \leq n$. Thus (3.7) is equivalent to $\left\{\left(*_{i}\right)\right\}_{1 \leq i \leq n}$ and the proof of our theorem is complete.

Set $\sum_{Y}=\sum_{i=1}^{n} y_{i}, \sum_{Y} \in Y$. The next result offers two general homogeneity criteria.

Proposition 3.3. If $\partial \in \operatorname{Der}_{1}^{+}$is of the form $\partial=\sum_{j \geq h} \partial_{j}$, with $\partial_{j} \in \operatorname{Der}_{1}^{j}$, then:

(i) $\partial$ is h-homogenous if and only if there is a filtered Lie isomorphism $v: \widehat{\mathbf{L}}_{X} \stackrel{\sim}{\longrightarrow} \widehat{\mathbf{L}}_{X}$ such that $\operatorname{gr}_{F}^{*}(v)=i d$ with respect to the canonical filtration $\left\{F_{k} \widehat{\mathbf{L}}_{X}\right\}_{k \geq 1}$ and $v(\partial Y) \subset\left\langle\left\langle\partial_{h} Y\right\rangle\right\rangle$, where $\langle\langle(\cdot)\rangle\rangle$ is the closed Lie ideal of $\widehat{\mathbf{L}}_{X}$ generated by $(\cdot)$.

(ii) Assuming that $\partial_{h} \sum_{Y}=0$ and that $\partial_{h}$ induces an injection $Y / \mathbf{Q}$. $\sum_{Y} \longrightarrow \mathbf{L}_{X}^{h+1}, \partial$ is h-homogenous if and only if there exist a filtered Lie isomorphism $v: \widehat{\mathbf{L}}_{X} \stackrel{\sim}{\longrightarrow} \widehat{\mathbf{L}}_{X}$ and a homogenous derivation $\partial_{h}^{\prime} \in$ $\operatorname{Der}_{1}^{h}$ satisfying $\partial_{h}^{\prime} \sum_{Y}=0$ and such that $v(\partial Y) \subset\left\langle\left\langle\partial_{h}^{\prime} Y\right\rangle\right\rangle$.

Proof. By definition $\partial$ is $h$-homogenous if and only if $u \partial=\partial_{h} u$, for some $u \in U$. For obvious degree reasons it is enough to check the above equality only on the free generators belonging to $Y$, which amounts to

$$
v\left(\partial y_{i}\right)=\partial_{h}\left(u y_{i}\right), \text { for } i=1, \ldots, n,
$$

where $v=u_{\mid \widehat{\mathbf{L}}_{X}}$. To say that $u \in U$ is equivalent (by the definition of $U$, see $\S 1$ ) to saying that $v$ is a filtered Lie automorphism of $\widehat{\mathbf{L}}_{X}$ satisfying $\operatorname{gr}_{F}^{*}(v)=i d$ and demanding the restriction of $u$ to $Y$ to be of the form

$$
u y_{i}=y_{i}+\sum_{j \geq 1} \varphi_{i}^{j}(x, y), \text { for } i=1, \ldots, n,
$$

with $\varphi_{i}^{j} \in \operatorname{ad}_{X}^{j}(Y)$, for all $j$.

These remarks give at once the "only if" part of the above statement (i). Conversely, knowing that $v(\partial Y) \subset\left\langle\left\langle\partial_{h} Y\right\rangle\right\rangle$ we infer equalities of the form

$$
v\left(\partial y_{i}\right)=\sum_{j \geq 0} \varphi_{i}^{j}\left(x, \partial_{h} y\right), \quad \text { for } \quad i=1, \ldots, n,
$$


with $\varphi_{i}^{j} \in \operatorname{ad}_{X}^{j}(Y)$ for $j \geq 0$ and where $\varphi_{i}^{j}\left(x, \partial_{h} y\right)$ denotes the result of the substitution $y_{l} \mapsto \partial_{h} y_{l}$, for every $l=1, \ldots, n$, in the Lie polynomial $\varphi_{i}^{j}(x, y)$. Using $\partial=\sum_{t \geq h} \partial_{t}$ and the unipotency of $v$, we obtain

$$
\partial_{h} y_{i}=\varphi_{i}^{0}\left(x, \partial_{h} y\right), \text { for all } i
$$

(by equating the lowest bracket degree terms of the preceding relations). Therefore we may suppose that $\varphi_{i}^{0}(x, y)=y_{i}$, for all $i$. Set then $u_{\mid \widehat{\mathbf{L}}_{X}}=v$ and $u y_{i}=\sum_{j \geq 0} \varphi_{i}^{j}(x, y)$, for $i=1, \ldots, n$, to obtain $u \in U$ with the property that ${ }^{u} \partial=\partial_{h}$, as required.

The "only if" part in the above statement (ii) follows from (i), with $\partial_{h}^{\prime}=$ $\partial_{h}$. For the converse implication, set $g=\operatorname{gr}_{F}^{1}(v)^{-1}: X \stackrel{\sim}{\longrightarrow} X$, denote by $\widehat{g} \in$ Aut $\widehat{\mathbf{L}}_{X}$ the completion of the (upper) degree zero Lie automorphism of $\mathbf{L}_{X}^{*}$ extending $g$, and write $v=\widehat{g}^{-1} u$, with $u \in$ Aut $\widehat{\mathbf{L}}_{X}$. Then $\operatorname{gr}_{F}^{1}(u)=i d$ by construction, whence $\operatorname{gr}_{F}^{*}(u)=i d$ ( map for any $u \in$ Aut $\widehat{\mathbf{L}}_{X}$ and $\operatorname{gr}_{F}^{*} \widehat{\mathbf{L}}_{X}=\mathbf{L}_{X}^{*}$ is generated in degree one). The hypothesis $v(\partial Y) \subset\left\langle\left\langle\partial_{h}^{\prime} Y\right\rangle\right\rangle$ readily implies that $u(\partial Y) \subset\left\langle\left\langle\widehat{g} \partial_{h}^{\prime} Y\right\rangle\right\rangle$. Due to the fact that the ideal $\left\langle\left\langle\widehat{g} \partial_{h}^{\prime} Y\right\rangle\right\rangle$ is homogenous and $u$ is unipotent, a lowest bracket degree argument as before reveals that necessarily $\partial_{h} Y \subset \widehat{g} \partial_{h}^{\prime} Y$. Whence we get a commutative diagram

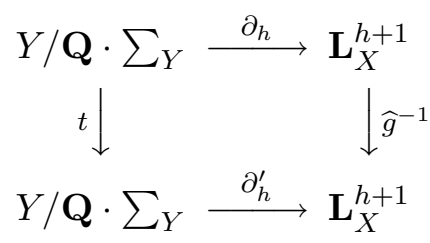

(for some linear map $t$ ).

The assumption on the injectivity of $\partial_{h}$ from the above diagram readily implies that $t$ is an isomorphism and therefore we actually have $\partial_{h} Y=\widehat{g} \partial_{h}^{\prime} Y$. Consequently $u(\partial Y) \subset\left\langle\left\langle\partial_{h} Y\right\rangle\right\rangle$ and we are done, by resorting to (i).

Remark 3.4. Let $K$ be a link having a Milnor system $\underline{l}$ such that $l_{i}^{(h)} \equiv 1$ modulo $\Gamma_{h} \mathbf{F}_{X}$, for all $i$ (for example $K=\widehat{\alpha}$, with $\alpha \in \Gamma_{h} P_{n}$, see Lemma 2.2). Set $\partial=\partial(\underline{l})$. It follows then from [22, Theorem 4.3 and (4.6)] that $\partial=\sum_{j \geq h} \partial_{j}$ and $\partial_{h} \sum_{Y}=0$. (In the case $K=\widehat{\alpha}$, with $\alpha \in \Gamma_{h} P_{n}$, this translates to $\sum_{i=1}^{n}\left[x_{i}, \bar{l}_{i}(\alpha)\right]=0$, see the proof of Corollary 2.8 (iii).) The other assumption made on $\partial_{h}$ in Proposition 3.3 (ii) is most natural, too. For example, if $h=1$ one may combine $[\mathbf{2 2},(4.7)]$ and $[\mathbf{1 7}, \S 6]$ to see that the map $\partial_{1}: Y / \mathbf{Q} \cdot \sum_{Y} \rightarrow \mathbf{L}_{X}^{2} \equiv \wedge^{2} X$ may be identified with the comultiplication $\Delta: H_{2} \rightarrow \wedge^{2} H_{1}$ (where $H_{*}=H_{*}\left(S^{3} \backslash K\right.$; Q )). It turns out that the injectivity of $\Delta$ characterizes the so-called generic links; see $[\mathbf{1}$, Example 4.3]. These enjoy several remarkable properties which have been 
studied in $[\mathbf{1}, \mathbf{2}, \mathbf{1 7}]$. For example all algebraic links (in the sense of $[\mathbf{2 0}$, $\S 10])$ are generic; see [1, Example 4.5].

The preceding proposition may be used to produce several interesting examples of homogenous links. For instance, denoting by $\sigma_{1}, \ldots, \sigma_{n-1}$ the standard generators of the full braid group $B_{n}$, see [3, p. 18], and setting $z_{n}=\left(\sigma_{1} \cdots \sigma_{n-1}\right)^{n}$, one knows that $z_{n}$ is a central element of $B_{n}$ which belongs to $P_{n}$, for $n \geq 2$, see $[\mathbf{3}, 1.8 .4]$. Also $f_{n}=\sigma_{n-1} \sigma_{n-2} \cdots \sigma_{1}^{2} \cdots \sigma_{n-2} \sigma_{n-1}$ $\in P_{n}, n \geq 2$, gives an interesting series of examples related to fibered links, see e.g., $[21]$.

Corollary 3.5. The pure braids $z_{n}, f_{n}$ and $a_{r s}$, where $1 \leq r<s \leq n$, and $n \geq 2$, are 1-homogenous.

Proof. The algebraic longitudes of the standard generators of $P_{n}, a_{r s}$, may be immediately read off from $[\mathbf{3},(1.15)]$ :

$$
\begin{cases}l_{i}\left(a_{r s}\right)=1, & \text { if } i<r \text { or } i>s, \\ l_{i}\left(a_{r s}\right)=\left(x_{r}, x_{s}\right), & \text { if } r<i<s, \\ l_{r}\left(a_{r s}\right)=x_{r} x_{s}, & \text { for } i=r \\ l_{s}\left(a_{r s}\right)=x_{r}, & \text { for } i=s\end{cases}
$$

Similarly, using the description of the Artin representation of the standard generators $\sigma_{i}$ of $B_{n}$, see $[\mathbf{3},(1.14)]$, it is straightforward to see that the longitudes of $f_{n}$ and $z_{n}$ are as follows (see e.g., $[\mathbf{2 1}, \mathbf{2 2}]$ ):

$$
\begin{cases}l_{i}\left(f_{n}\right)=x_{1} \cdots x_{n-1} x_{n}, & \text { for } i<n \\ l_{n}\left(f_{n}\right)=x_{1} \cdots x_{n-1}, & \text { for } i=n\end{cases}
$$

and

$$
l_{i}\left(z_{n}\right)=x_{1} \cdots x_{n} \cdot x_{i}^{-1}, \text { for } 1 \leq i \leq n .
$$

This gives at once the following formulae for $\partial_{1}$, by abelianization (since $\left.\rho \Gamma_{2} \mathbf{F}_{X} \subset \widehat{\mathbf{L}}_{\bar{X}}^{\geq 2}\right)$ :

$$
\left\{\begin{array}{l}
\partial_{1}\left(a_{r s}\right) y_{i}=0, \text { for } i \neq r, s \\
\partial_{1}\left(a_{r s}\right) y_{r}=\left[x_{r}, x_{s}\right] \\
\partial_{1}\left(a_{r s}\right) y_{s}=\left[x_{s}, x_{r}\right]
\end{array}\right.
$$

$$
\left\{\begin{array}{l}
\partial_{1}\left(f_{n}\right) y_{i}=\left[x_{i}, x_{n}\right], \text { for } i<n, \\
\partial_{1}\left(f_{n}\right) y_{n}=\left[x_{n}, \Sigma_{X}\right]
\end{array}\right.
$$

(where $\left.\sum_{X}=: \sum_{i=1}^{n} x_{i}, \sum_{X} \in X\right)$, and

$$
\partial_{1}\left(z_{n}\right) y_{i}=\left[x_{i}, \Sigma_{X}\right], \text { for } 1 \leq i \leq n .
$$

The basic Campbell-Hausdorff trick of our proof is based on the following standard remark (see [14] and also [22, Lemma 2.5]):

$$
\left\langle\left\langle\left(w_{1}, w_{1}^{\prime}\right), \ldots,\left(w_{m}, w_{m}^{\prime}\right)\right\rangle\right\rangle=\left\langle\left\langle\left[w_{1}, w_{1}^{\prime}\right], \ldots,\left[w_{m}, w_{m}^{\prime}\right]\right\rangle\right\rangle,
$$


for any elements $w_{i}, w_{i}^{\prime} \in \widehat{\mathbf{L}}_{X}$, where $[\cdot, \cdot]$ is the Lie algebra commutator and $(\cdot, \cdot)$ is the $\mathrm{CH}$-group commutator.

The proof for $\alpha=a_{r s}$ and $\alpha=f_{n}$ is a direct consequence of Proposition $3.3(\mathrm{i})$, taking $v=i d$. To verify that $\partial(\alpha) y_{i}=\left[x_{i}, \rho l_{i}(\alpha)\right] \equiv 0$ modulo $\left\langle\left\langle\partial_{1}(\alpha) y_{j}=\left[x_{j}, \bar{l}_{j}(\alpha)\right] \quad \mid 1 \leq j \leq n\right\rangle\right\rangle$, for all $1 \leq i \leq n$, it suffices by the previous remark to see that $\left(x_{i}, \rho l_{i}(\alpha)\right) \equiv \rho(1)$ modulo $\left\langle\left\langle\left(x_{j}, \bar{l}_{j}(\alpha)\right)\right| 1 \leq\right.$ $j \leq n\rangle\rangle$, for all $1 \leq i \leq n$. This amounts to making a routine check, for both $\alpha=a_{r s}$ and $\alpha=f_{n}$. Namely we have to see that in the $C H$-group of $\widehat{\mathbf{L}}_{X}$ the relations $\left(x_{j}, \bar{l}_{j}(\alpha)\right)=1$, for all $j$, imply the relations $\left(x_{i}, \rho l_{i}(\alpha)\right)=1$, for all $i$, given the classical correspondence between closed ideals and normal $\mathrm{CH}$-subgroups of complete Lie algebras [14].

Finally the proof for $\alpha=z_{n}$ uses Proposition 3.3 (ii). We shall take $\partial_{1}^{\prime}=\partial_{1}\left(f_{n}\right)$ and note that $\partial_{1}\left(z_{n}\right) \sum_{Y}=0$ and $\partial_{1}\left(f_{n}\right) \sum_{Y}=0$ (see Remark 3.4). The injectivity condition for $\partial_{1}\left(z_{n}\right): Y / \mathbf{Q} \cdot \sum_{Y} \longrightarrow \mathbf{L}_{X}^{2}$ immediately follows from the commutation properties of a free Lie algebra, via $\left(3.10^{\prime}\right)$. To construct $v$, set

$$
\left\{\begin{array}{l}
v x_{i}=x_{i}, \text { for } i<n, \\
v x_{n}=\left(x_{1} \cdots x_{n-1}\right)^{-1} x_{n} .
\end{array}\right.
$$

This defines a filtered Lie isomorphism $v: \widehat{\mathbf{L}}_{X} \stackrel{\sim}{\rightarrow} \widehat{\mathbf{L}}_{X}$, which necessarily is also a group automorphism of the associated $C H$-group of $\widehat{\mathbf{L}}_{X}$ [14]. As in the previous two cases, to check that $v\left(\partial\left(z_{n}\right) y_{i}\right)=\left[v x_{i}, v \rho l_{i}\left(z_{n}\right)\right] \equiv 0$ modulo $\left\langle\left\langle\partial_{1}^{\prime} y_{j}=\left[x_{j}, \bar{l}_{j}\left(f_{n}\right)\right] \mid 1 \leq j \leq n\right\rangle\right\rangle$, for all $1 \leq i \leq n$, and thus finish our proof, we may replace in the above congruences all Lie brackets by $\mathrm{CH}$-commutators and then replace $\left\langle\left\langle\partial_{1}^{\prime} y_{j} \mid 1 \leq j \leq n\right\rangle\right\rangle$ by the normal $\mathrm{CH}$ subgroup generated by $\left(x_{j}, x_{n}\right), 1 \leq j<n$. Resorting to (3.11) and (3.10), it is straightforward to see that the relations $\left(x_{j}, x_{n}\right)=1$, for $1 \leq j<n$, imply the relations $\left(v x_{i}, v \rho l_{i}\left(z_{n}\right)\right)=1$, for all $i$, which completes the proof.

Corollary 3.6. All algebraic links (in the sense of $[\mathbf{2 0}, \S 10]$ ) are 1-homogenous.

Proof. The key property of algebraic link complements $S^{3} \backslash K$ is the fact that they are formal spaces, in the sense of rational homotopy theory [25], as shown in [6, Theorem 4.2]. Setting $G=\pi_{1}\left(S^{3} \backslash K\right)$, it is well-known that this implies the existence of a filtered Lie algebra isomorphism between the Malcev Lie algebra $G \otimes \mathbf{Q}$ and the (completed) holonomy Lie algebra $\widehat{L}_{S^{3} \backslash K}$; see e.g., [13] for details and references on $G \otimes \mathbf{Q}$ and $L$.

The Malcev Lie algebra of $G$ is by definition $G \otimes \mathbf{Q}=: \lim _{k}\left(\left(G / \Gamma_{k} G\right) \otimes \mathbf{Q}\right)$. Here $\left(G / \Gamma_{k} G\right) \otimes \mathbf{Q}$ is the $\mathbf{Q}$-Lie algebra form of the Malcev completion of the nilpotent group $G / \Gamma_{k} G$ and the Lie algebra $G \otimes \mathbf{Q}$ is endowed with the canonical (complete) inverse limit filtration. Let $\underline{l}$ be any Milnor system of longitudes of an arbitrary $n$-link $K$. As pointed out in [22, Remark 2.9], 
there is a filtered Lie isomorphism

$$
G \otimes \mathbf{Q} \cong \lim _{k}\left(\widehat{\mathbf{L}}_{X} /\left(\left\langle\left\langle\partial^{(k)} Y\right\rangle\right\rangle+\widehat{\mathbf{L}}_{\bar{X}}^{\geq k}\right)\right),
$$

where $\partial^{(k)}=\partial\left(\underline{l}^{(k)}\right)$, with $\underline{l}^{(k)}=\left\{l_{i}^{(k)}\right\}_{1 \leq i \leq n}$, for each $k$. It is immediate to see that we may replace in $(3.12) \partial^{(k)}$ by $\partial=\partial(\underline{l})$, for every $k$, to get a filtered Lie isomorphism

$$
G \otimes \mathbf{Q} \cong \widehat{\mathbf{L}}_{X} /\langle\langle\partial Y\rangle\rangle
$$

(where the filtration of the quotient is induced by the canonical filtration $\left.\left\{F_{s} \widehat{\mathbf{L}}_{X}=\widehat{\mathbf{L}}_{\bar{X}}^{\geq s}\right\}_{s}\right)$.

On the other hand, we have shown in $[\mathbf{2 2},(4.7)]$ how to compute $\partial_{1}$ in terms of linking numbers. This gives, via $[\mathbf{1 7}, \S 6]$, another filtered Lie isomorphism, valid for any link $K$,

$$
\widehat{L}_{S^{3} \backslash K} \cong \widehat{\mathbf{L}}_{X} /\left\langle\left\langle\partial_{1} Y\right\rangle\right\rangle
$$

(where the quotient carries again the filtration induced from $\widehat{\mathbf{L}}_{X}$ ).

We may now finish our proof by resorting to Proposition 3.3 (ii). We may take $\partial_{1}^{\prime}=\partial_{1}$, recalling that in general one has $\partial_{1} \sum_{Y}=0$ and that $\partial_{1}$ of an algebraic link induces an injection $Y / \mathbf{Q} \cdot \sum_{Y} \rightarrow \mathbf{L}_{X}^{2}$; see Remark 3.4. As for the filtered Lie isomorphism $v$, it may be obtained in a standard way by combining (3.13) and (3.14).

Set $E=\widehat{\mathbf{L}}_{X} /\langle\langle\partial Y\rangle\rangle$ and $E_{1}=\widehat{\mathbf{L}}_{X} /\left\langle\left\langle\partial_{1} Y\right\rangle\right\rangle$. We have a filtered Lie isomorphism $E \stackrel{\sim}{\rightarrow} E_{1}$, which may be lifted by freeness to a filtered Lie morphism $v: \widehat{\mathbf{L}}_{X} \rightarrow \widehat{\mathbf{L}}_{X}$, with the property that $v(\partial Y) \subset\left\langle\left\langle\partial_{1} Y\right\rangle\right\rangle$. Since plainly both canonical projections, $\widehat{\mathbf{L}}_{X} \rightarrow E$ and $\widehat{\mathbf{L}}_{X} \rightarrow E_{1}$, induce isomorphisms at the $\operatorname{gr}_{F}^{1}$-level, we infer that $\operatorname{gr}_{F}^{1}(v): X \stackrel{\sim}{\rightarrow} X$ is an isomorphism. This implies that $\operatorname{gr}_{F}^{*}(v)$ is an isomorphism, since $\operatorname{gr}_{F}^{*}\left(\widehat{\mathbf{L}}_{X}\right)=\mathbf{L}_{X}^{*}$ and $v$ is a Lie map. We get inductively that $v: \widehat{\mathbf{L}}_{X} / F_{k} \stackrel{\sim}{\rightarrow} \widehat{\mathbf{L}}_{X} / F_{k}$ is an isomorphism, for all $k$, therefore $v: \widehat{\mathbf{L}}_{X} \stackrel{\sim}{\rightarrow} \widehat{\mathbf{L}}_{X}$ is an isomorphism, as needed, by the completeness of $\widehat{\mathbf{L}}_{X}$.

\section{Some homogenous $C H$-tests.}

In this section we are going to reformulate on examples our key homogenous $C H$-test $\left\{\left(*_{i}\right)\right\}_{1 \leq i \leq n}$ (see Theorem 3.2 (ii)) in terms of the graded Lie algebra $\operatorname{gr}_{\Gamma}^{*} P_{n}$. As a first step we shall get rid of ad $\left(x_{i}\right)$ in front of $\bar{l}_{i}\left(\bar{\beta}-\overline{\beta^{\prime}}\right)$ from $\left(*_{i}\right)$.

To be precise, we are going to look at the property :

there exists $\theta \in \operatorname{Der}^{k-1} \mathbf{L}_{X}^{*}$ such that

$$
\left[x_{i}, \bar{l}_{i}(\bar{\gamma})\right] \equiv\left[x_{i}, \theta \bar{l}_{i}(\bar{\alpha})\right]+\left[\theta x_{i}, \bar{l}_{i}(\bar{\alpha})\right]
$$




$$
\text { modulo }\left\langle\left[x_{j}, \bar{l}_{j}(\bar{\alpha})\right] \mid 1 \leq j \leq n\right\rangle
$$

for each $i=1, \ldots, n$

of a given $\gamma \in \Gamma_{k} P_{n}(k \geq 2)$, where $\alpha$ is one of the 1-homogenous braids $z_{n}$ or $a_{r s}$ of Corollary 3.5.

Beginning with $\alpha=z_{n}$, it will be convenient to consider, for any fixed $j$, $1 \leq j \leq n$, the graded Lie algebra map $\pi_{j}: \mathbf{L}^{*}\left(x_{1}, \ldots, x_{n}\right) \longrightarrow \mathbf{L}^{*}\left(x_{s} \mid s \neq\right.$ $j$ ) given on the free generators by

$$
\left\{\begin{array}{l}
\pi_{j} x_{i}=x_{i}, \text { for } i \neq j \\
\pi_{j} x_{j}=-\sum_{i \neq j} x_{i}
\end{array}\right.
$$

Lemma 4.1. The above conditions $\left\{\left(*_{i}^{\prime}\right)\right\}_{1 \leq i \leq n}$ are equivalent for $\alpha=z_{n}$ with the conditions

$$
\bar{l}_{i}(\bar{\gamma}) \equiv \bar{l}_{j}(\bar{\gamma}) \text { modulo }\left\langle\left[x_{t}, \Sigma_{X}\right] \mid 1 \leq t \leq n\right\rangle \text {, for all } 1 \leq i, j \leq n
$$

or equivalently (for any fixed $j, 1 \leq j \leq n$ )

$$
\pi_{j} \bar{l}_{i}(\bar{\gamma})=\pi_{j} \bar{l}_{j}(\bar{\gamma}), \text { for all } i \neq j \text {. }
$$

Proof. Setting $I=\left\langle\left[x_{t}, \bar{l}_{t}\left(z_{n}\right)\right] \mid 1 \leq t \leq n\right\rangle$ it is immediate to see that $I=$ $\left\langle\left[x_{t}, \sum_{X}\right] \mid 1 \leq t \leq n\right\rangle$ (look at $(3.10)$ and $\left(3.10^{\prime}\right)$ ). Since $\bar{l}_{i}\left(z_{n}\right)=\sum_{X}-x_{i}$, for every $i$, the right-hand side of $\left(*_{i}^{\prime}\right)$ equals (modulo $I$ )

$$
\left[x_{i}, \theta \Sigma_{X}\right]-\left[x_{i}, \theta x_{i}\right]-\left[\theta x_{i}, x_{i}\right]=\left[x_{i}, \theta \Sigma_{X}\right]-\theta\left[x_{i}, x_{i}\right]=\left[x_{i}, \theta \Sigma_{X}\right] .
$$

Therefore the conditions $\left\{\left(*_{i}^{\prime}\right)\right\}_{1 \leq i \leq n}$ just say that $\left[x_{i}, \bar{l}_{i}(\bar{\gamma})-\eta\right] \equiv 0$ modulo $I$, with $i=1, \ldots, n$, for some $\eta \in \overline{\mathbf{L}}_{X}^{k}$.

Notice that for any fixed $j, \mathbf{L}_{X}^{*} / I$ is isomorphic to $\mathbf{L}^{*}\left(x_{s} \mid s \neq j\right) \times \mathbf{Q} \cdot \sum_{X}$, the graded Lie direct product of $\mathbf{L}^{*}\left(x_{s} \mid s \neq j\right)$ and the one-dimensional abelian graded Lie algebra concentrated in degree one $\mathbf{Q} \cdot \sum_{X}$, and the map $\pi_{j}$ defined in (4.1) represents the projection of $\mathbf{L}_{X}^{*} / I$ onto the first factor. We may now invoke again the commutation properties of free graded Lie algebras $[\mathbf{1 6}$, p. 328] (remembering that $k \geq 2$ ) to get all the desired conclusions of the lemma.

A similar argument will do the job in the other case $\left(\alpha=a_{r s}\right)$.

Lemma 4.2. The above conditions $\left\{\left(*_{i}^{\prime}\right)\right\}_{1 \leq i \leq n}$ are equivalent for $\alpha=a_{r s}$ with

$$
\bar{l}_{i}(\bar{\gamma}) \equiv 0 \text { modulo }\left\langle\left[x_{r}, x_{s}\right]\right\rangle \text {, for all } i \neq r, s .
$$

Proof. Set $I=:\left\langle\left[x_{r}, x_{s}\right]\right\rangle=\left\langle\left[x_{j}, \bar{l}_{j}\left(a_{r s}\right)\right] \mid 1 \leq j \leq n\right\rangle$, see (3.8) and $\left(3.8^{\prime}\right)$. Then the conditions $\left\{\left(*_{i}^{\prime}\right)\right\}_{1 \leq i \leq n}$ take the form

$$
\left\{\begin{array}{lll}
{\left[x_{i}, \bar{l}_{i}(\bar{\gamma})\right]} & \equiv_{I} & 0, \text { for } i \neq r, s \\
{\left[x_{r}, \bar{l}_{r}(\bar{\gamma})\right]} & \equiv_{I} & {\left[x_{r}, \theta x_{s}\right]+\left[\theta x_{r}, x_{s}\right],} \\
{\left[x_{s}, \bar{l}_{s}(\bar{\gamma})\right]} & \equiv_{I} & {\left[x_{s}, \theta x_{r}\right]+\left[\theta x_{s}, x_{r}\right],}
\end{array}\right.
$$


for some $\theta \in \operatorname{Der}^{k-1} \mathbf{L}_{X}^{*}$. Taking into account that $\sum_{i=1}^{n}\left[x_{i}, \bar{l}_{i}(\bar{\gamma})\right]=0$ (see Remark 3.4) the last of the above conditions follows from the others. On the other hand the preceding one obviously is always fulfilled, by defining $\theta x_{s}=\bar{l}_{r}(\bar{\gamma})$ and $\theta x_{j}=0$ for $j \neq s$. Therefore it suffices to show that $\left[x_{i}, l\right] \equiv_{I} 0$ implies $l \equiv_{I} 0$, for any $l \in \mathbf{L}_{X}^{k}, k \geq 2$, and for any $i \neq r, s$, in order to finish our proof. For a fixed such $i$ it is enough to establish the corresponding commutation property for the universal enveloping algebra $U\left(\mathbf{L}_{X}^{*} / I\right)$, which is isomorphic to $\left(\mathbf{T}^{*}\left(x_{j} \mid j \neq i\right) /\left\langle x_{r} x_{s}-x_{s} x_{r}\right\rangle\right) \amalg \mathbf{T}^{*}\left(x_{i}\right)$, graded by tensor degree. Namely it suffices to show that the centralizer of $x_{i}$ equals $\mathbf{T}^{*}\left(x_{i}\right)$, which in turn can be easily verified, given the free product structure of the algebra $U\left(\mathbf{L}_{X}^{*} / I\right)$.

Now we may take a second step and express everything only in terms of $\operatorname{gr}_{\Gamma}^{*} P_{n}$.

Proposition 4.3. If $\gamma \in \Gamma_{k} P_{n}(k \geq 2)$ is such that $\bar{\gamma}$ belongs to the graded Lie subalgebra of $\operatorname{gr}_{\Gamma}^{*} P_{n}$ generated by $\left\{\bar{a}_{r s} \mid r, s \neq j\right\}$, for some $j, 1 \leq$ $j \leq n$ (e.g., if $\left.\gamma \in \Gamma_{k} P_{n-1}\right)$, then $\gamma$ meets the requirements of the $\mathrm{CH}$-test $\left\{\left(*_{i}^{\prime}\right)\right\}_{i \leq i \leq n}$ corresponding to $\alpha=z_{n}$ if and only if $\bar{\gamma}=0$, where $\bar{\gamma} \in \operatorname{gr}_{\Gamma}^{k} P_{n}$ denotes as usual the class of $\gamma$ modulo $\Gamma_{k+1} P_{n}$.

Proof. Let $E_{j}^{*} \subset \operatorname{gr}_{\Gamma}^{*} P_{n}$ be the graded Lie subalgebra generated by $\left\{\bar{a}_{r s} \mid\right.$ $r, s \neq j\}$. The result will follow easily from the following claim:

For any $e \in E_{j}^{t}(t \geq 1)$ one has

$$
\bar{l}_{j}(e)=0
$$

and

$$
\bar{l}_{i}(e) \in \mathbf{L}^{t}\left(x_{p} \mid p \neq j\right), \text { for all } i \neq j \text {. }
$$

Granting the above claim, we may finish our argument as follows. We know from Lemma 4.1 that the $C H$-test $\left\{\left(*_{i}^{\prime}\right)\right\}_{1 \leq i \leq n}$ is equivalent with the fact that $\pi_{j} \bar{l}_{i}(\bar{\gamma})=\pi_{j} \bar{l}_{j}(\bar{\gamma})$, for all $i \neq j$. By hypothesis $\bar{\gamma} \in E_{j}^{k}$, hence $\pi_{j} \bar{l}_{j}(\bar{\gamma})=0($ see $(4.2))$ and $\pi_{j} \bar{l}_{i}(\bar{\gamma})=\bar{l}_{i}(\bar{\gamma})$, for all $i \neq j$ (see (4.3) and (4.1)). Therefore our test is equivalent with $\bar{l}_{i}(\bar{\gamma})=0$ for all $i, 1 \leq i \leq n$, and this happens if and only if $\bar{\gamma}=0$, as asserted, according to Theorem 2.7.

As far as our above two claims are concerned, we may induct on $t$. At step $t=1$ both (4.2) and (4.3) follow at once from Lemma 2.5. For the induction step, assume (4.2)-(4.3) to hold for $e, e^{\prime} \in E_{j}^{*}$. We are going to show that they also hold for $\left[e, e^{\prime}\right]$ by using Lemma 2.4 to write

$$
\bar{l}_{i}\left[e, e^{\prime}\right]=\left[\bar{l}_{i}(e), \bar{l}_{i}\left(e^{\prime}\right)\right]+\widetilde{e}\left(\bar{l}_{i}\left(e^{\prime}\right)\right)-\widetilde{e}^{\prime}\left(\bar{l}_{i}(e)\right) \text {, for } 1 \leq i \leq n .
$$

This already gives the result claimed in (4.2), for $i=j$. For $i \neq j$ we may use again the above formula together with the induction hypotheses on $e$ and 
$e^{\prime}$. To complete the proof of our second claim (4.3) we note that for any $f \in$ $\operatorname{gr}_{\Gamma}^{*} P_{n}$ having the property that $\bar{l}_{i}(f) \in \mathbf{L}^{*}\left(x_{p} \mid p \neq j\right)$, for all $1 \leq i \leq n$, the associated derivation $\tilde{f} \in \operatorname{Der}{ }^{*} \mathbf{L}\left(x_{1}, \ldots, x_{n}\right)$ defined in Lemma 2.4 leaves invariant the Lie subalgebra $\mathbf{L}^{*}\left(x_{p} \mid p \neq j\right) \subset \mathbf{L}^{*}\left(x_{1}, \ldots, x_{n}\right)$. This property in turn needs to be checked only for the generators $x_{p}(p \neq j)$, due to the fact that $\tilde{f}$ is a Lie derivation. One has $\widetilde{f} x_{p}=\left[x_{p}, \bar{l}_{p}(f)\right]$, by construction, and this is clearly an element belonging to the desired subalgebra, by our assumptions on $f$, for any $p \neq j$.

Concerning the graded Lie algebra structure of $\operatorname{gr}_{\Gamma}^{*} P_{n}$, one knows for example (see $[\mathbf{3}, \S 1.4]$ ) that the split exact sequence $(2.5)$ exhibits $P_{n}$ as a semidirect product, $P_{n}=\mathbf{F}\left(a_{r n} \mid 1 \leq r<n\right) \times P_{n-1}$. The structure map is given by the Artin representation $P_{n-1} \longrightarrow$ Aut $\mathbf{F}\left(a_{r n} \mid r<n\right.$ ) (after an obvious change of notation for the generators of the free group). Accordingly [7] $\operatorname{gr}_{\Gamma}^{*} P_{n}$ splits as a semidirect graded Lie product

$$
\operatorname{gr}_{\Gamma}^{*} P_{n}=\mathbf{L}^{*}\left(\bar{a}_{r n} \mid r<n\right)(\mathbf{Z}) \times \operatorname{gr}_{\Gamma}^{*} P_{n-1} .
$$

Here the adjoint representation of $\operatorname{gr}_{\Gamma}^{*} P_{n-1}$ in the free Lie algebra equals by construction the graded Lie algebra map

$$
\begin{aligned}
\lambda & =-J: \operatorname{gr}_{\Gamma}^{*} P_{n-1} \longrightarrow \operatorname{Der}^{*}\left(\mathbf{L}\left(\bar{a}_{r n} \mid r<n\right)(\mathbf{Z})\right) \\
& \equiv \operatorname{Der}^{*}\left(\mathbf{L}\left(x_{1}, \ldots, x_{n-1}\right)(\mathbf{Z})\right)
\end{aligned}
$$

(where $J$ is Johnson's homomorphism, as in Proposition 2.1); see the proof of Lemma 2.4. We recall that if $\gamma \in \Gamma_{k} P_{n-1}$ then $\lambda(\bar{\gamma})=\widetilde{\gamma}$, where

$$
\widetilde{\gamma} x_{i}=\left[x_{i}, \bar{l}_{i}(\gamma)\right] \text {, for } i=1, \ldots, n-1 .
$$

The simplest nontrivial example occurs for $n=3$, where the above discussion implies that

$$
\operatorname{gr}_{\Gamma}^{*} P_{3}=\mathbf{L}^{*}\left(a_{1}, a_{2}\right)(\mathbf{Z}) \times(\mathbf{Z} \cdot a)
$$

(where we have denoted for future convenience $\overline{a_{r 3}}$ by $a_{r}$, for $r=1,2$, and $\overline{a_{12}}$ by $a$ ); here $\mathbf{Z} \cdot a$ is a free rank one abelian $\mathbf{Z}$-Lie algebra concentrated in degree one and

$$
\left[a, a_{1}\right]=\left[a_{2}, a\right]=\left[a_{1}, a_{2}\right]
$$

(see Lemma 2.5).

For concrete computations one may use standard Hall bases of free Lie algebras, see e.g., $[\mathbf{1 6}]$; for example $\left\{\left[a_{1}, a_{2}\right]\right\}$ is a $\mathbf{Z}$-basis of $\mathbf{L}^{2}\left(a_{1}, a_{2}\right)(\mathbf{Z})$, $\left\{A_{1}=:\left[a_{1},\left[a_{1}, a_{2}\right]\right], A_{2}=:\left[a_{2},\left[a_{1}, a_{2}\right]\right]\right\}$ is a $\mathbf{Z}$-basis of $\mathbf{L}^{3}\left(a_{1}, a_{2}\right)(\mathbf{Z}) \ldots$

The simplest nontrivial homogenous $C H$-test corresponding to $\alpha=a_{r s}$ occurs for $n=3$ and $k=3$ and it may be ultimately rephrased within $\operatorname{gr}_{\Gamma}^{*} P_{3}$ as follows. 
Proposition 4.4. For a given $\gamma \in \Gamma_{3} P_{3}$, set

$$
\bar{\gamma}=c_{1}\left[a_{1},\left[a_{1}, a_{2}\right]\right]+c_{2}\left[a_{2},\left[a_{1}, a_{2}\right]\right] \text {, with } c_{1,2} \in \mathbf{Z}
$$

(where $\bar{\gamma} \in \operatorname{gr}_{\Gamma}^{3} P_{3}$ stands as usual for the class of $\gamma$ modulo $\left.\Gamma_{4} P_{3}\right)$. Then $\gamma$ satisfies the conditions $\left\{\left(*_{i}^{\prime}\right)\right\}_{1 \leq i \leq 3}$ corresponding to $\alpha=a_{12} \in P_{3}$ if and only if $c_{1}=c_{2}$.

Proof. By Lemma 4.2, all we have to show is that $\bar{l}_{3}(\bar{\gamma}) \equiv 0 \bmod \left\langle\left[x_{1}, x_{2}\right]\right\rangle$ if and only if $c_{1}=c_{2}$.

By a repeated application of Lemmas 2.4 and 2.5 we find that

$$
\begin{aligned}
& \bar{l}_{3}\left(\left[a_{1},\left[a_{1}, a_{2}\right]\right]\right)=\left[x_{1},\left[x_{1}, x_{2}\right]\right]+\left[\left[x_{1}, x_{3}\right], x_{2}\right]-\left[x_{1},\left[x_{2}, x_{3}\right]\right], \\
& \bar{l}_{3}\left(\left[a_{2},\left[a_{1}, a_{2}\right]\right]\right)=\left[x_{2},\left[x_{1}, x_{2}\right]\right]+\left[x_{1},\left[x_{2}, x_{3}\right]\right]-\left[x_{2},\left[x_{3}, x_{1}\right]\right] .
\end{aligned}
$$

Computing modulo $I=:\left\langle\left[x_{1}, x_{2}\right]\right\rangle$ we arrive at

$$
\begin{aligned}
& \bar{l}_{3}\left(\left[a_{1},\left[a_{1}, a_{2}\right]\right]\right) \quad \equiv_{I} 2 \cdot\left[\left[x_{2}, x_{3}\right], x_{1}\right], \\
& \bar{l}_{3}\left(\left[a_{2},\left[a_{1}, a_{2}\right]\right]\right) \quad \equiv_{I}-2 \cdot\left[\left[x_{2}, x_{3}\right], x_{1}\right] .
\end{aligned}
$$

The additive property of Lemma 2.3 and the passage to the enveloping algebra $U\left(\mathbf{L}_{X} / I\right)=\mathbf{Q}\left[x_{1}, x_{2}\right] \coprod \mathbf{Q}\left[x_{3}\right]$ allow us to reformulate the conditions $\left\{\left(*_{i}^{\prime}\right)\right\}_{1 \leq i \leq 3}$ as follows

$$
2\left(c_{1}-c_{2}\right) \cdot\left[\left[x_{2}, x_{3}\right], x_{1}\right]=0 \text { in } U\left(\mathbf{L}_{X} / I\right) .
$$

This is equivalent with $c_{1}=c_{2}$, as asserted, since one may expand the brackets and easily see that $\left[\left[x_{2}, x_{3}\right], x_{1}\right]$ is a nonzero element of the free product of polynomial algebras, $\mathbf{Q}\left[x_{1}, x_{2}\right] \amalg \mathbf{Q}\left[x_{3}\right]$.

\section{Unordered isotopy classes of pure links and $\Sigma_{n}$-actions.}

As is well-known [3], the conjugation in $B_{n}$ does not change the oriented isotopy type of the Artin braid closure. For pure closed braids, which come naturally equipped also with an ordering of their components (corresponding to the labels of the strings), one has the following more precise statement:

Given any $b, b^{\prime} \in P_{n}$, their Artin closures $\widehat{b}$ and $\widehat{b^{\prime}}$ are oriented (not necessarily ordered) isotopic if and only if there exists $\sigma \in B_{n}$, belonging to some fixed complete set of representatives for the cosets of $P_{n}$, such that $\widehat{\sigma} \vec{b}$ and $\widehat{b^{\prime}}$ are oriented (and ordered) isotopic links.

Thus our $C H$-invariants $\left\{p^{(k)}\right\}_{k \geq 2}$ may be used to detect unordered oriented isotopy types of pure links, via the conjugation action of $B_{n}$ on $P_{n}$ and the induced action of $\Sigma_{n}$ on the graded Lie algebra $\operatorname{gr}_{\Gamma}^{*} P_{n}$, see Lemma 2.6.

On the other hand, let us recall from [22, Proposition 2.8] another natural action of $\Sigma_{n}$, by bigraded Lie algebra automorphisms, on the bigraded Lie algebra of derivations $\operatorname{Der}_{*}^{*} \mathbf{L}(X \oplus Y)$ described in Section 1. The group 
Aut $\mathbf{L}_{*}^{*}(X \oplus Y)$ of bihomogenous Lie algebra automorphisms, which clearly acts on the bigraded Lie algebra $\operatorname{Der}_{*}^{*} \mathbf{L}(X \oplus Y)$ by conjugation, may be identified with $G L(X) \times G L(Y)$, by taking the restrictions of automorphisms to the free generators. The above mentioned action of $\Sigma_{n}$ on Der ${ }_{*}^{*}$ is defined by restriction, via the following product representation of $\Sigma_{n}$ in $G L(X) \times$ $G L(Y)$, given for any $g \in \Sigma_{n}$ by

$$
\begin{cases}g x_{i}=x_{g i}, & \text { for } i=1, \ldots, n, \\ g y_{i}=y_{g i}, & \text { for } i=1, \ldots, n .\end{cases}
$$

We begin with the following $\Sigma_{n}$-equivariant appendix of our homogenous $\mathrm{CH}$-test (Theorem 3.2).

\section{Lemma 5.1.}

(i) The map $\Lambda: \operatorname{gr}_{\Gamma}^{*} P_{n} \longrightarrow \operatorname{Der}_{1}^{*} \mathbf{L}(X \oplus Y)$ constructed in (2.7) is equivariant with respect to the above $\Sigma_{n}$-actions.

(ii) For any $\alpha \in \Gamma_{h} P_{n}$ and $g \in \Sigma_{n}$ one has

$$
{ }^{g} \partial_{h}(\alpha)=\partial_{h}(\alpha) \text { if and only if } g \bar{\alpha}=\bar{\alpha}
$$

(where $g \bar{\alpha}$ denotes the conjugation action of $g$ on the class of $\alpha, \bar{\alpha} \in$ $\left.\operatorname{gr}_{\Gamma}^{h} P_{n}\right)$.

(iii) For any $\alpha \in \Gamma_{h} P_{n}$ set $H=\left\{g \in \Sigma_{n} \mid{ }^{g} \partial_{h}(\alpha)=\partial_{h}(\alpha)\right\}$. Then the map

$$
\operatorname{ad}\left(\partial_{h}(\alpha)\right): \operatorname{Der}_{0}^{*} \mathbf{L}(X \oplus Y) \longrightarrow \operatorname{Der}_{1}^{*+h} \mathbf{L}(X \oplus Y)
$$

is $H$-equivariant.

Proof. The first basic equivariant property is a consequence of Lemma 2.6, which may be used to compute, for any $g \in \Sigma_{n}, \gamma \in \Gamma_{s} P_{n}$, and $1 \leq i \leq n$

$$
\Lambda(g \bar{\gamma})\left(y_{i}\right)=\left[x_{i}, \bar{l}_{i}(g \bar{\gamma})\right]=\left[x_{i}, g \bar{l}_{g^{-1} i}(\bar{\gamma})\right] .
$$

On the other hand, we get, using the definition of $\Lambda$ (see (2.7)) and (5.1) above

$$
{ }^{g} \Lambda(\bar{\gamma})\left(y_{i}\right)=g \Lambda(\bar{\gamma})\left(y_{g^{-1} i}\right)=g\left[x_{g^{-1} i}, \bar{l}_{g^{-1} i}(\bar{\gamma})\right]=\left[x_{i}, g \bar{l}_{g^{-1} i}(\bar{\gamma})\right] .
$$

The second assertion of the lemma is then a corollary of the first, due to the fact that $\partial_{h}(\alpha)=\Lambda(\bar{\alpha})$ (see the proof of Corollary 2.8 (iii)), in conjunction with Theorem 2.7, which implies that $\Lambda$ is injective. (For the injectivity of $\Lambda$ one also needs to use the commutation properties of a free Lie algebra, together with the normalization condition (1.2) in degree $*=1$.)

Finally, the last statement (iii) may be checked by a routine application of the fact that $\Sigma_{n}$ acts on $\operatorname{Der}_{*}^{*} \mathbf{L}(X \oplus Y)$ by Lie algebra automorphisms.

For practical purposes one may compute the $\Sigma_{n}$-action on $\operatorname{gr}_{\Gamma}^{*} P_{n}$ by resorting to the graded Lie algebra structure. This is a folk statement, whose relevant details are going to be recorded for the reader's convenience. 
The semidirect graded Lie splitting described in (4.4)-(4.5) together with Lemma 2.5 may be used to argue inductively on $n$. Thus it can be shown that $\operatorname{gr}_{\Gamma}^{k} P_{n}$ is a finitely generated free abelian group, for all $k \geq 1$. In particular the component $\operatorname{gr}_{\Gamma}^{1} P_{n}$ admits as a $\mathbf{Z}$-basis the set $\left\{\bar{a}_{r s} \mid 1 \leq r<s \leq n\right\}$ (which obviously may be identified with the set of 2-element subsets of $\{1, \ldots, n\}$, by associating to $\bar{a}_{r s}$ the subset $\left.\{r, s\}\right)$. Finally, in this way one may also obtain a system of defining relations between the graded Lie algebra generators of $\operatorname{gr}_{\Gamma}^{*} P_{n},\left\{\bar{a}_{r s}\right\}_{1 \leq r<s \leq n}$. It is given by the relations $\left(4.5^{\prime}\right)$ corresponding to every 3 distinct indices $1 \leq r, s, t \leq n$,

$$
\left[\bar{a}_{r s}, \bar{a}_{r t}\right]=\left[\bar{a}_{s t}, \bar{a}_{r s}\right]=\left[\bar{a}_{r t}, \bar{a}_{s t}\right],
$$

together with the commutation relations

$$
\left[\bar{a}_{r s}, \bar{a}_{t u}\right]=0,
$$

for every 4 distinct indices $1 \leq r, s, t, u \leq n$. The $\Sigma_{n}$-module structure of $\operatorname{gr}_{\Gamma}^{*} P_{n}$ may then be handled as in the lemma below.

\section{Lemma 5.2.}

(i) The $\Sigma_{n}$-module $\operatorname{gr}_{\Gamma}^{1} P_{n}$ is isomorphic to the free $\mathbf{Z}$-module generated by the $\Sigma_{n}$-set of 2 -element subsets of $\{1, \ldots, n\}$, endowed with the obvious $\Sigma_{n}$-action, for every $n$.

(ii) The above property, together with $\left(4.4^{\prime}\right)$ and $\left(4.5^{\prime}\right)$, suffice to describe the $\Sigma_{3}$-action on $\operatorname{gr}_{\Gamma}^{k} P_{3}$, for all $k$.

Proof. Remember that we have identified $\bar{a}_{r s} \in \operatorname{gr}_{\Gamma}^{1} P_{n}$ with $\{r, s\} \subset\{1$, $\ldots, n\}$. For the proof of (i), it will be plainly enough to show that $g \cdot \bar{a}_{r, s}=$ $\bar{a}_{g r, g s}$, for any $g \in \Sigma_{n}$ (with the convention that $\bar{a}_{u v}=: \bar{a}_{v u}$, if $1 \leq v<$ $u \leq n)$. Equivalently, we must show that $\bar{l}_{i}\left(g \cdot \bar{a}_{r, s}\right)=\bar{l}_{i}\left(\bar{a}_{g r, g s}\right)$, for all $i$; see Theorem 2.7 and Lemma 2.3. This we may do by an easy computation which uses Lemmas 2.6 and 2.5.

We may formulate the above statement (ii) more precisely, for $k=2$. Namely we claim that $\operatorname{gr}_{\Gamma}^{2} P_{3}$ is the free rank one signature $\mathbf{Z}$-module of $\Sigma_{3}$. To see this it is enough (see $\left(4.4^{\prime}\right)$ ) to verify that $\tau_{1}\left[a_{1}, a_{2}\right]=\tau_{2}\left[a_{1}, a_{2}\right]=$ $-\left[a_{1}, a_{2}\right]$; here we have denoted by $\tau_{i} \in \Sigma_{n}$ the transposition $(i, i+1)$, for $1 \leq i<n$. To make the above computation, one may use the fact that $\Sigma_{n}$ acts on $\operatorname{gr}_{\Gamma}^{*} P_{n}$ by graded Lie algebra automorphisms, Lemma 5.2 (i) and the defining relations $\left(4.5^{\prime}\right)$. For example,

$$
\tau_{1}\left[a_{1}, a_{2}\right]=\left[\tau_{1} \bar{a}_{13}, \tau_{1} \bar{a}_{23}\right]=\left[\bar{a}_{23}, \bar{a}_{13}\right]=-\left[a_{1}, a_{2}\right],
$$

and similarly

$$
\tau_{2}\left[a_{1}, a_{2}\right]=\left[\bar{a}_{12}, \bar{a}_{23}\right]=\left[a, a_{2}\right]=-\left[a_{1}, a_{2}\right] .
$$

Inductively, we have to compute the action of $g \in \Sigma_{3}$ on a basic commutator of the form $\left[c, c^{\prime}\right]$, with $c \in \mathbf{L}^{s}\left(a_{1}, a_{2}\right)(\mathbf{Z}), c^{\prime} \in \mathbf{L}^{t}\left(a_{1}, a_{2}\right)(\mathbf{Z}), s+t=k \geq 3$, and $s \leq t$. We have to distinguish two cases. When $s \geq 2, g\left[c, c^{\prime}\right]=\left[g c, g c^{\prime}\right]$, 
with $g c \in \mathbf{L}^{s}\left(a_{1}, a_{2}\right)(\mathbf{Z})$ and $g c^{\prime} \in \mathbf{L}^{t}\left(a_{1}, a_{2}\right)(\mathbf{Z})$ already computed. Thus we just have to rewrite $\left[g c, g c^{\prime}\right]$ as a linear combination of basic commutators in $\mathbf{L}^{k}\left(a_{1}, a_{2}\right)(\mathbf{Z})$, see e.g., [16]. If $s=1$ then necessarily $t \geq 2$ (since $k \geq 3$ ), hence $g c^{\prime} \in \mathbf{L}^{t}\left(a_{1}, a_{2}\right)(\mathbf{Z})$. On the other hand, $g c$ is also already computed and belongs to $\left\{a_{1}, a_{2}, a\right\}$, by property (i). If $g c=a_{r}(r=1,2)$, then we are done, as before. Finally if $g c=a$ then $\left[a, g c^{\prime}\right] \in \mathbf{L}^{k}\left(a_{1}, a_{2}\right)(\mathbf{Z})$ may be computed by Jacobi with the aid of the relations $\left(4.5^{\prime}\right)$, which completes our inductive step.

Example 5.3 $(k=3)$. The action of $\Sigma_{3}$ on $\operatorname{gr}_{\Gamma}^{3} P_{3}$ is given by

$$
\begin{array}{ll}
\tau_{1} A_{1}=-A_{2}, & \tau_{1} A_{2}=-A_{1}, \\
\tau_{2} A_{1}=A_{1}+A_{2}, & \tau_{2} A_{2}=-A_{2},
\end{array}
$$

where $\left\{A_{1}=\left[a_{1},\left[a_{1}, a_{2}\right]\right], A_{2}=\left[a_{2},\left[a_{1}, a_{2}\right]\right]\right\}$ is the Hall basis of $\operatorname{gr}_{\Gamma}^{3} P_{3}=$ $\mathbf{L}^{3}\left(a_{1}, a_{2}\right)(\mathbf{Z})$ and $\tau_{i}$ is the transposition $(i, i+1)$, for $i=1,2$. Indeed,

$$
\left\{\begin{array}{l}
\tau_{1} A_{1}=-\left[a_{2},\left[a_{1}, a_{2}\right]\right]=-A_{2}, \\
\tau_{1} A_{2}=-\left[a_{1},\left[a_{1}, a_{2}\right]\right]=-A_{1},
\end{array}\right.
$$

(by the results of Lemma 5.2 for $k=1,2$ ) and

$$
\left\{\begin{array}{l}
\tau_{2} A_{1}=-\left[a,\left[a_{1}, a_{2}\right]\right]=-\left[\left[a, a_{1}\right], a_{2}\right]-\left[a_{1},\left[a, a_{2}\right]\right]=A_{1}+A_{2}, \\
\tau_{2} A_{2}=-\left[a_{2},\left[a_{1}, a_{2}\right]\right]=-A_{2}
\end{array}\right.
$$

(by the results of Lemma 5.2 for $k=1,2$, the Jacobi identity and the relations $\left.\left(4.5^{\prime}\right)\right)$.

\section{6. $C H$-invariants versus Vassiliev and other link invariants.}

We have pointed out in [22] some similarities and differences between our $\mathrm{CH}$-invariants and Milnor's $\bar{\mu}$-invariants. In this section we are going to present a similar comparison with finite type invariants, on examples. Vassiliev's invariants are more general than the $\mathrm{CH}$-invariants (which are of finite type, in a certain sense; see [15]) and more powerful than the $\mathrm{CH}$ invariants (which fail to distinguish knots; see $[\mathbf{2 2}, \S 3]$ ). On the other hand, the $\mathrm{CH}$-approach has the advantage to offer a more specific useful concrete tool for many-component links, as we shall soon see. We will examine a series of three classes of examples. As in the previous sections, we are going to look at the Artin closures of pairs of pure braids of the form $b=\beta \alpha$ and $b^{\prime}=\beta^{\prime} \alpha$, with $\alpha \in P_{n}$ and $\beta, \beta^{\prime} \in \Gamma_{k} P_{n}$, where $k \geq 2$.

The basic motivation is provided by Stanford's result [24, Theorem 1], which says that $\widehat{b}$ and $\widehat{b^{\prime}}$ have the same Vassiliev invariants of order less than or equal to $k-1$. We have seen, in Corollary 2.8, that a similar phenomenon occurs for the $C H$-invariants, namely that $p^{(k)}(b)=p^{(k)}\left(b^{\prime}\right)$. Stanford also showed how to use his preceding result to obtain infinite families of distinct links, sharing the same Vassiliev invariants, up to order $k-1$, with an 
arbitrary given link $K$; see [24, Theorem 2]. We begin by constructing similar (less general but simpler) classes of examples, which are faithfully detected by $p^{(k+1)}$. The point is that the $C H$-theory offers a concrete $p^{(k+1)}$ test, whenever $p^{(k)}(K)=p^{(k)}\left(K^{\prime}\right)$, while it seems to be a difficult job to find a specific higher order Vassiliev invariant to distinguish $K$ from $K^{\prime}$.

We shall take $\alpha$ equal to the central braid $z_{n} \in P_{n}(n>3), \beta=\gamma^{m}$ and $\beta^{\prime}=\gamma^{t}(m, t \geq 0)$, where $\gamma \in \Gamma_{k} P_{n}$ is such that $\bar{\gamma} \in \operatorname{gr}_{\Gamma}^{k} P_{n}$ is a nonzero element belonging to the graded Lie subalgebra generated by $\left\{\bar{a}_{r s} \mid r, s \neq j\right\}$ for some $j, 1 \leq j \leq n$ (one may use for example any nonzero $\bar{\gamma} \in \operatorname{gr}_{\Gamma}^{k} P_{n-1}$ ), as in Proposition 4.3.

Proposition 6.1. The pure links $\left\{\widehat{\gamma^{m} z_{n}}\right\}_{m \geq 0}$ have the same Vassiliev invariants of order less than or equal to $k-1$ and the same $\bar{\mu}$-invariants of arbitrarily high order, for any $\gamma \in \Gamma_{k} P_{n}(k \geq 2)$. If $\bar{\gamma} \in \operatorname{gr}_{\Gamma}^{k} P_{n}$ is as above then $\widehat{\gamma^{m} z_{n}}$ and $\widehat{\gamma^{t} z_{n}}$ are oriented isotopic if and only if $m=t$. The isotopy type is detected by $p^{(k+1)}$.

Proof. The assertion on Milnor's $\bar{\mu}$-invariants of the oriented and ordered links $\widehat{\gamma^{m} z_{n}}$ may be easily proved as follows. As is well-known [19] (see also [22]) the first $\bar{\mu}$-invariants $\bar{\mu}_{i j}(1 \leq i, j \leq n)$ have trivial indeterminacy $\left(\bar{\mu}_{i j}=\mu_{i j}\right)$ and may be computed by abelianization from the algebraic longitudes. More precisely, $\bar{l}_{i}(\alpha)=\sum_{j=1}^{n} \bar{\mu}_{i j}(\widehat{\alpha}) x_{j} \in \operatorname{gr}{ }_{\Gamma}^{1} \mathbf{F}_{X}$, for any $\alpha \in P_{n}$. By Lemma 2.3 and (3.10) we thus see that $\bar{\mu}_{i j}\left(\widehat{\gamma^{m} z_{n}}\right)=1$ for all $i \neq j$ and $\bar{\mu}_{i i}\left(\widehat{\gamma^{m} z_{n}}\right)=0$ for all $i$. Since in general $\bar{\mu}_{I}$, where $I=\left(i_{1}, \ldots, i_{s}\right)$, and $1 \leq i_{1}, \ldots, i_{s} \leq n$, is defined only modulo $\Delta_{I} \cdot \mathbf{Z}$, where $\Delta_{I}$ is the greatest common divisor of certain $\mu$-integers $\mu_{J}$, where the multiindex $J$ is shorter than $I$ (see [19]), it is straightforward to infer that all the other $\bar{\mu}$-invariants are trivial: $\bar{\mu}_{I}\left(\widehat{\gamma^{m} z_{n}}\right)=0$ for all $s>2$.

On the other hand, the Artin closures of $\gamma^{m} z_{n}$ and $\gamma^{t} z_{n}$ are oriented (not necessarily ordered) isotopic if and only if the closures of ${ }^{\sigma}\left(\gamma^{m} z_{n}\right)$ and $\gamma^{t} z_{n}$ are oriented and ordered isotopic for some $\sigma \in B_{n}$, as recalled in Section 5. For the rest of the proof it will be then enough to show that if $p^{(k+1)}\left(\sigma\left(\gamma^{m}\right) z_{n}\right)=p^{(k+1)}\left(\gamma^{t} z_{n}\right)$ for some $\sigma \in B_{n}$ then necessarily $m=t$. By Theorem 3.2 and Corollary 3.5 the equality of the above $p^{(k+1)}$ invariants is equivalent with the fact that the derivation $\Lambda(m \cdot g \bar{\gamma}-t \cdot \bar{\gamma})$ belongs to $\operatorname{Im}$ ad $\left(\partial_{1}\left(z_{n}\right)\right.$ ) for some $g \in \Sigma_{n}$ (where the action of $\Sigma_{n}$ on $\operatorname{gr}_{\Gamma}^{*} P_{n}$ is induced by the conjugation action of $B_{n}$, as in Lemma 2.6), since $z_{n}$ is 1-homogenous. Since $z_{n}$ is a central element of $B_{n}$, we infer from Lemma 5.1 (ii)-(iii) that ad $\left(\partial_{1}\left(z_{n}\right)\right)$ is a $\Sigma_{n}$-equivariant map, and consequently $\operatorname{Der}{ }_{1}^{k} \mathbf{L}(X \oplus Y) / \operatorname{Im}$ ad $\left(\partial_{1}\left(z_{n}\right)\right)$ is a (finite-dimensional) $\Sigma_{n}$-vector space. Therefore the above $p^{(k+1)}$-test may be rephrased as saying that $m \cdot g \Lambda(\bar{\gamma})=t \cdot \Lambda(\bar{\gamma})$ (where the equality is understood to take place in Der ${ }_{1}^{k} \mathbf{L}(X \oplus Y) / \operatorname{Im}$ ad $\left.\left(\partial_{1}\left(z_{n}\right)\right)\right)$, for some $g \in \Sigma_{n}$; use the equivariant Lemma 
5.1 (i). Since $\bar{\gamma} \neq 0$ by hypothesis, the homogenous $C H$-test from Proposition 4.3 and Theorem 3.2 (ii) may be invoked to infer that $\Lambda(\bar{\gamma})$ represents a nonzero vector modulo $\operatorname{Im} \operatorname{ad}\left(\partial_{1}\left(z_{n}\right)\right)$.

If $m=0$ this readily implies $t=0$ and if $m \neq 0$ we know that $t / m$ is an eigenvalue of the finite-order linear map induced by $g$ on the quotient, hence $t= \pm m$, which implies that actually $t=m$, since $m, t \geq 0$.

Clearly the first interesting examples of pure links arise from $P_{3}$. While we had to assume, in the preceding result, that $n>3$, our next result shows that the $\mathrm{CH}$-method may be used efficiently for pure links on 3 strands, too. Consider for example $b=\beta \alpha$ and $b^{\prime}=\beta^{-1} \alpha$, where $\alpha$ is the standard generator $a_{12} \in P_{3}$ and $\beta \in \Gamma_{3} P_{3}$. Set $\bar{\beta}=c_{1} A_{1}+c_{2} A_{2}$, where $A_{i}=$ $\left[a_{i},\left[a_{1}, a_{2}\right]\right]$ and $a_{i}=\overline{a_{i 3}}$, for $i=1,2$, as in Proposition 4.4.

Proposition 6.2. The pure links $\widehat{\beta \alpha}$ have the same sublinks (up to oriented isotopy) and are link-homotopic, for any $\beta \in \Gamma_{3} P_{3}$. If $c_{1} \neq c_{2}$ then $\widehat{\beta \alpha}$ and $\widehat{\beta^{-1}} \alpha$ are not oriented isotopic, the difference between them being picked up by $p^{(4)}$.

Proof. The first assertion is immediate. Deleting the $i$ th strand corresponds to applying the canonical projection $p_{i}: P_{3} \longrightarrow P_{2}$, for $1 \leq i \leq 3$, see [3]. Since $P_{2}$ is abelian $p_{i}(\beta \alpha)=p_{i}\left(a_{12}\right)$, for all $i$, therefore the 2-sublinks are independent of $\beta$ (and are either trivial or Hopf links). The passage from link isotopy to link homotopy corresponds to the canonical projection $p: P_{3} \longrightarrow \mathcal{H}(3)$ and one knows that $\Gamma_{3} \mathcal{H}(3)=0$, see $[\mathbf{9}, 1.12]$, whence the claim on link-homotopy. (Actually both the above properties are a consequence of the fact that the links $\widehat{\beta \alpha}$ share the same $\bar{\mu}$-invariants.)

As in the previous proof, we shall conclude by showing that $p^{(4)}\left({ }^{\sigma} b\right)=$ $p^{(4)}\left(b^{\prime}\right)$ for some $\sigma \in B_{3}$ implies that $c_{1}=c_{2}$. Firstly $p^{(4)}\left({ }^{\sigma} b\right)=p^{(4)}\left(b^{\prime}\right)$ implies that $p^{(2)}\left({ }^{\sigma} b\right)=p^{(2)}\left(b^{\prime}\right)[\mathbf{2 2}$, Theorem A (ii)]. The equality of the $p^{(2)}$-invariants translates in turn to the fact that $\bar{l}_{i}(\overline{\sigma b})=\bar{l}_{i}\left(\bar{b}^{\prime}\right)$, for all $i$ (see [22, Remark 2.7]), due to the relation between abelianized longitudes and Milnor's $\bar{\mu}_{i j}$-invariants, explained in the preceding proof. Consequently, (see Theorem 2.7) $g \bar{a}_{12}=\bar{a}_{12}$, with $g=\nu\left(\sigma^{-1}\right)$, as in Lemma 2.6. By the description of the $\Sigma_{3}$-module $\operatorname{gr}_{\Gamma}^{1} P_{3}$ recorded in Lemma 5.2 (i), $g$ must be either 1 or the transposition $\tau_{1}=(12)$. Therefore either $\sigma=1$, or $\sigma=\sigma_{1}$ and ${ }^{\sigma} b={ }^{\sigma} \beta \cdot \alpha$, since $a_{12}=\sigma_{1}^{2}$; see $[3]$.

The 1-homogeneity of $\alpha$ (Corollary 3.5) implies, via Theorem 3.2 (ii) plus the homogenous $C H$-test of Proposition 4.4, that $p^{(4)}\left({ }^{\sigma} b\right)=p^{(4)}\left(b^{\prime}\right)$ if and only if one has $c_{1}(g \bar{\beta}+\bar{\beta})=c_{2}(g \bar{\beta}+\bar{\beta})$, where $\bar{\gamma}=c_{1}(\bar{\gamma}) A_{1}+c_{2}(\bar{\gamma}) A_{2}$ is the expression of any given element $\bar{\gamma} \in \operatorname{gr}_{\Gamma}^{3} P_{3}$ with respect to the basis $\left\{A_{1}, A_{2}\right\}$, as usual. 
If $g=1$ this gives that $c_{1}(\bar{\beta})=c_{2}(\bar{\beta})$, as desired. For $g=\tau_{1}$ one has that $c_{1}(g \bar{\beta})=-c_{2}(\bar{\beta})$ and $c_{2}(g \bar{\beta})=-c_{1}(\bar{\beta})$; see Example 5.3. Hence $c_{1}(g \bar{\beta}+\bar{\beta})=c_{2}(g \bar{\beta}+\bar{\beta})$ if and only if $c_{1}(\bar{\beta})=c_{2}(\bar{\beta})$, as stated.

It was noticed in $[\mathbf{1 5}]$ that the $C H$-invariants, like the $\bar{\mu}$ - invariants, cannot be determined by quantum invariants. Our last result exhibits examples having the same HOMFLY polynomial and at the same time equal $\bar{\mu}$-invariants of arbitrarily high order, which may be handled by homogenous $C H$-tests. We shall consider pairs of the form $b=\beta z_{n}$ and $b^{\prime}=\beta^{-1} z_{n}$, $\left(b, b^{\prime} \in P_{n}\right)$, with $\beta \in \Gamma_{3} P_{3}$. Set $\bar{\beta}=c_{1} A_{1}+c_{2} A_{2}$, as in the previous example.

Proposition 6.3. The pure $n$-links $\widehat{\beta z_{n}}$ and $\widehat{\beta^{-1} z_{n}}$ (with $n \geq 3$ ) have the same HOMFLY polynomial, the same $\bar{\mu}$-invariants and are link-homotopic, for any $\beta \in \Gamma_{3} P_{3}$. If $c_{1} \neq c_{2}, c_{1} \neq 0$ and $c_{2} \neq 0$, then they are distinguished by $p^{(4)}$, up to oriented isotopy, for $n>6$.

Proof. As before, we are going to prove that if $p^{(4)}\left({ }^{\sigma} b\right)=p^{(4)}\left(b^{\prime}\right)$ for some $\sigma \in B_{n}$ then $c_{1}=c_{2}$ or $c_{1}=0$ or $c_{2}=0$, for $n>6$. The fact that $p^{(4)}\left(\sigma(\beta) z_{n}\right)=p^{(4)}\left(\beta^{-1} z_{n}\right)$ for some $\sigma \in B_{n}$ may be reformulated as follows: $\pi_{n} \bar{l}_{i}(g \bar{\beta}+\bar{\beta})=\pi_{n} \bar{l}_{j}(g \bar{\beta}+\bar{\beta})$, for all $1 \leq i \neq j \leq n$, for some $g \in \Sigma_{n}$; see Theorem 3.2 (ii) and Lemma 4.1. By Lemma 2.6 this condition in turn may be rewritten as follows

$$
\pi_{n} g \bar{l}_{g^{-1} i}(\bar{\beta})+\pi_{n} \bar{l}_{i}(\bar{\beta})=\pi_{n} g \bar{l}_{g^{-1} j}(\bar{\beta})+\pi_{n} \bar{l}_{j}(\bar{\beta})
$$

(for all $1 \leq i \neq j \leq n$ ), for some $g \in \Sigma_{n}$. We are going first to infer that $g$ must leave invariant the subset $\{1,2,3\}$.

To this end, let us start by noting that $\bar{l}_{i}(\bar{\beta}) \in \mathbf{L}\left(x_{1}, x_{2}, x_{3}\right)(\mathbf{Z})$, for $i \leq 3$, and $\bar{l}_{i}(\bar{\beta})=0$, for $i>3$, since $\beta \in P_{3}$ and consequently $x_{i} \beta \in \mathbf{F}\left(x_{1}, x_{2}, x_{3}\right)$, for $i \leq 3$, and $x_{i} \beta=x_{i}$, for $i>3$; see the definitions (1.1)-(1.3). On the other hand it follows immediately from (4.6), given the definition of $\pi_{3}$ (see (4.1)), that $\pi_{3} \bar{l}_{3}(\bar{\beta})=c_{1}\left[x_{2},\left[x_{1}, x_{2}\right]\right]+c_{2}\left[x_{1},\left[x_{1}, x_{2}\right]\right]$. A similar iteration of Lemmas 2.4 and 2.5 readily gives that $\pi_{3} \bar{l}_{1}(\bar{\beta})=\pi_{3} \bar{l}_{2}(\bar{\beta})=\pi_{3} \bar{l}_{3}(\bar{\beta})$. If there is some $i \leq 3$ such that $g^{-1} i>3$ and at the same time there is some $j>3$ such that $g^{-1} j>3$, then the equality $(*)$ implies that $\pi_{n} \bar{l}_{i}(\bar{\beta})=0$. Given that $\bar{l}_{i}(\bar{\beta}) \in \mathbf{L}\left(x_{1}, x_{2}, x_{3}\right)(\mathbf{Z})$ for $i \leq 3$, we may conclude by (4.1) that necessarily $\bar{l}_{i}(\bar{\beta})=0$, if $n>3$. Hence $\pi_{3} \bar{l}_{i}(\bar{\beta})=0$, and this implies that $c_{1}=c_{2}=0$, by our previous computation. Thus we infer that either $g(\{1,2,3\})=\{1,2,3\}$ or $g^{-1}(\{4, \ldots, n\}) \subset\{1,2,3\}$; in the second case we get that $n \leq 6$, a contradiction. Denote by $h \in \Sigma_{3} \subset \Sigma_{n}$ the restriction of $g$ to $\{1,2,3\}$ (i.e., $h i=g i$ for $i \leq 3$ and $h i=i$ for $i>3$ ). We deduce from the above discussion that the relations $(*)$ continue to hold also for $h$. Therefore $h \bar{\beta}+\bar{\beta} \in \operatorname{gr}_{\Gamma}^{3} P_{3} \subset \operatorname{gr}{ }_{\Gamma}^{3} P_{n}$ meets the requirements of the homogenous $C H$-test of Lemma 4.1. For $n>3$ this implies, via Proposition 4.3, that $h \bar{\beta}=-\bar{\beta}$, for some $h \in \Sigma_{3}$. 
The explicit description of the $\Sigma_{3}$-module $\operatorname{gr}_{\Gamma}^{3} P_{3}$ (see Example 5.3) leads then immediately to the desired conclusion: $c_{1}=c_{2}$ or $c_{1}=0$ or $c_{2}=0$ (corresponding to $h=(12)$ or $h=(23)$ or $h=(13)$ ).

The equality of the $\bar{\mu}$-invariants follows from Proposition 6.1 and the assertion on link-homotopy is proved as in Proposition 6.2. The proof of the coincidence of the HOMFLY polynomials is based on an idea of Birman [4] and uses the general machinery developed in [11] (see also [5]). One knows that for each $n$ there exists a representation of $B_{n}$ into a finite-dimensional C-algebra $\mathcal{B}_{n}, \rho_{n}: B_{n} \longrightarrow \mathcal{B}_{n}$, inducing a surjection $\mathbf{C}\left[B_{n}\right] \longrightarrow \mathcal{B}_{n}$. Moreover there is a trace (a $\mathbf{C}$-linear map vanishing on the algebra commutators) $\tau_{n}: \mathcal{B}_{n} \longrightarrow$ C. Here $\mathcal{B}_{n}, \rho_{n}$ and $\tau_{n}$ depend on two complex parameters and have the property that: Given any $b, b^{\prime} \in B_{n}$ such that $e(b)=e\left(b^{\prime}\right)$ (where $e$ stands for the exponent sum with respect to the standard generators $\sigma_{1}, \ldots, \sigma_{n-1}$ of $B_{n}$ ), the HOMFLY polynomials of their Artin closures $\widehat{b}, \widehat{b^{\prime}}$ will coincide as soon as $\tau_{n} \rho_{n}(b)=\tau_{n} \rho_{n}\left(b^{\prime}\right)$. See [11], [5]. In our case $e(b)=e\left(b^{\prime}\right)=e\left(z_{n}\right)$, since $\beta \in \Gamma_{2} B_{n}$.

Next one knows [11] that generically $\mathcal{B}_{n}$ is semisimple (it is isomorphic to $\left.\mathbf{C}\left[\Sigma_{n}\right]\right)$. Therefore it splits as a product of full matrix algebras, $\mathcal{B}_{n}=$ $\prod_{i} M\left(d_{i} ; \mathbf{C}\right)$, and the restriction of $\tau_{n}$ to each $M\left(d_{i} ; \mathbf{C}\right)$ is the usual matrix trace, up to a normalization factor. It will be thus enough to check that $\operatorname{tr} \pi_{i} \rho_{n}(b)=\operatorname{tr} \pi_{i} \rho_{n}\left(b^{\prime}\right)$, for all $i$, where $\pi_{i}: \mathcal{B}_{n} \longrightarrow M\left(d_{i} ; \mathbf{C}\right)$ denotes the canonical projection. Since $z_{n}$ is central in $\mathbf{C}\left[B_{n}\right], \pi_{i} \rho_{n}\left(z_{n}\right)$ will be a scalar, for all $i$. Thus we are left with proving that $\operatorname{tr} \pi_{i} \rho_{n}(\beta)=\operatorname{tr} \pi_{i} \rho_{n}\left(\beta^{-1}\right)$ for all $i$, if $\beta \in \Gamma_{2} B_{3}$ and $n \geq 3$. Since one also has group inclusions $B_{m} \subset B_{n}$ and algebra maps $\mathcal{B}_{m} \longrightarrow \mathcal{B}_{n}$ commuting with the above representations $\rho_{m}$ and $\rho_{n}$, for $m \leq n$, it will plainly suffice to check the above equality of traces for $n=3$.

By the classical representation theory of $\Sigma_{3}$ there are three matrix representations of $B_{3}$ to be considered; see [11]. For two of them $d_{i}=1$ and consequently $\pi_{i} \rho_{3}(\beta)=\pi_{i} \rho_{3}\left(\beta^{-1}\right)=1$, since $\beta \in \Gamma_{2} B_{3}$. For the other one $d_{i}=2$, whence again $\operatorname{tr} \pi_{i} \rho_{3}(\beta)=\operatorname{tr} \pi_{i} \rho_{3}(\beta)^{-1}$. The last equality follows from elementary matrix theory, since $\pi_{i} \rho_{3}(\beta) \in S L(2 ; \mathbf{C})$ because $\beta \in \Gamma_{2} B_{3}$.

Acknowledgement. This work has been done while the author was visiting the University of Lille, whose support and hospitality are gratefully acknowledged.

\section{References}

[1] B. Berceanu and S. Papadima, Cohomologically generic 2-complexes and 3dimensional Poincaré complexes, Math. Ann., 298(3) (1994), 457-480. 
[2] _ Moduli spaces for generic low-dimensional complexes, J. Pure Appl. Alg., 95 (1994), 1-25.

[3] J. Birman, Braids, links and mapping class groups, Annals of Math. Studies, 82, Princeton Univ. Press, Princeton, 1974.

[4] _ On the Jones polynomial of closed 3-braids, Invent. Math., 81(2) (1985), 287-294.

[5] J. Birman and H. Wenzl, Braids, link polynomials and a new algebra, Trans. Amer. Math. Soc., 313(1) (1989), 249-273.

[6] A. Durfee and R. Hain, Mixed Hodge structures on the homotopy of links, Math. Ann., 280 (1988), 69-83.

[7] M. Falk and R. Randell, The lower central series of a fiber-type arrangement, Invent. Math., 82 (1985), 77-88.

[8] P. Freyd, D. Yetter, J. Hoste, W.B.R. Lickorish, K. Millet and A. Ocneanu, A new polynomial invariant of knots and links, Bull. Amer. Math. Soc., 12 (1985), 239-246.

[9] N. Habegger and X.-S. Lin, The classification of links up to link-homotopy, Journ. of the Amer. Math. Soc., 3(2) (1990), 389-419.

[10] D. Johnson, An abelian quotient of the mapping class group $\mathcal{T}_{g}$, Math. Ann., 249 (1980), 225-242.

[11] V.F.R. Jones, Hecke algebra representations of braid groups and link polynomials, Ann. of Math., 126 (1987), 335-388.

[12] L. Kaloujnine, Sur quelques propriétés des groupes d'automorphismes d'un groupe abstrait (généralisation d'un théorème de M. Ph. Hall), C. R. Acad. Sci., 231 (1950), 400-402.

[13] T. Kohno, Série de Poincaré-Koszul associée aux groupes de tresses pures, Invent. Math., 82 (1985), 57-75.

[14] M. Lazard, Sur les groupes nilpotents et les anneaux de Lie, Ann. Ec. Norm. Sup., 71 (1954), 101-190.

[15] X.-S. Lin, Finite type link invariants and the non-invertibility of links, Math. Research Letters, 3 (1996), 405-417.

[16] W. Magnus, A. Karrass and D. Solitar, Combinatorial group theory, Pure and Appl. Math., 13, Interscience, New York, 1966.

[17] M. Markl and S. Papadima, Homotopy Lie algebras and fundamental groups via deformation theory, Ann. Inst. Fourier, 42(4) (1992), 905-935.

[18] _ Moduli spaces for fundamental groups and link invariants derived from the lower central series, Manuscripta Math., 81 (1993), 225-242.

[19] J. Milnor, Isotopy of links, in 'Algebraic Geometry and Topology: A Symposium in Honor of S. Lefschetz' (Princeton Univ. Press, Princeton, 1957), 280-306.

[20] _ Singular points of complex hypersurfaces, Annals of Math. Studies, 61, Princeton Univ. Press, Princeton, 1968.

[21] S. Moran, The mathematical theory of knots and braids. An introduction, Mathematics Studies, 82, North-Holland, Amsterdam, 1983.

[22] S. Papadima, Campbell-Hausdorff invariants of links, Proc. London Math. Soc., 75(3) (1997), 641-670.

[23] J.-P. Serre, Lie algebras and Lie groups, Benjamin, New York, 1965. 
[24] T. Stanford, Braid commutators and Vassiliev invariants, Pacific J. of Math., 174 (1996), 269-276.

[25] D. Tanré, Homotopie rationnelle: Modèles de Chen, Quillen, Sullivan, Lect. Notes in Math., 1025, Springer-Verlag, 1983.

Received June 16, 1998.

Institute of Mathematics of the Romanian Academy

P.O. Box 1-764, RO-70700

BuCHAREST

ROMANIA

E-mail address: spapadim@stoilow.imar.ro 